\title{
Redox-Sensing Under Hypochlorite Stress and Infection Conditions by the Rrf2-Family Repressor HypR in Staphylococcus aureus
}

\author{
Vu Van Loi,, Tobias Busche,,2 Karsten Tedin,, Jörg Bernhardt, Jan Wollenhaupt, ${ }^{5}$ Nguyen Thi Thu Huyen, \\ Christoph Weise, Jörn Kalinowski, Markus C. Wahl, Marcus Fulde, and Haike Antelmann ${ }^{1}$
}

\begin{abstract}
Aims: Staphylococcus aureus is a major human pathogen and has to cope with reactive oxygen and chlorine species (ROS, RCS) during infections, which requires efficient protection mechanisms to avoid destruction. Here, we have investigated the changes in the RNA-seq transcriptome by the strong oxidant sodium hypochlorite $(\mathrm{NaOCl})$ in S. aureus USA300 to identify novel redox-sensing mechanisms that provide protection under infection conditions. Results: $\mathrm{NaOCl}$ stress caused an oxidative stress response in $S$. aureus as indicated by the induction of the PerR, QsrR, HrcA, and SigmaB regulons in the RNA-seq transcriptome. The hypR-merA (USA300HOU_0588-87) operon was most strongly upregulated under $\mathrm{NaOCl}$ stress, which encodes for the Rrf2-family regulator HypR and the pyridine nucleotide disulfide reductase MerA. We have characterized HypR as a novel redox-sensitive repressor that controls MerA expression and directly senses and responds to $\mathrm{NaOCl}$ and diamide stress via a thiol-based mechanism in S. aureus. Mutational analysis identified Cys33 and the conserved Cys99 as essential for $\mathrm{NaOCl}$ sensing, while Cys99 is also important for repressor activity of HypR in vivo. The redox-sensing mechanism of HypR involves Cys33-Cys99 intersubunit disulfide formation by $\mathrm{NaOCl}$ stress both in vitro and in vivo. Moreover, the HypR-controlled flavin disulfide reductase MerA was shown to protect $S$. aureus against $\mathrm{NaOCl}$ stress and increased survival in J774A.1 macrophage infection assays.

Conclusion and Innovation: Here, we identified a new member of the widespread Rrf2 family as redox sensor of $\mathrm{NaOCl}$ stress in $S$. aureus that uses a thiol/disulfide switch to regulate defense mechanisms against the oxidative burst under infections in S. aureus. Antioxid. Redox Signal. 29, 615-636.
\end{abstract}

Keywords: Staphylococcus aureus, Rrf2, redox-sensing regulator, hypochlorite stress

\section{Introduction}

$S$ TAPHYLOCOCCUS AUREUS IS not only a common commensal bacterium but also a major pathogen that accounts for $40 \%$ of nosocomial infections in humans (31). S. aureus can cause local wound infections and also severe life-threatening infections, such as septicemia, endocarditis, necrotizing pneu- monia, catheter-associated infections, and osteomyelitis ( 3 , $12,60)$. A major problem to combat $S$. aureus infections is the increasing prevalence of multiple antibiotic-resistant isolates, such as methicillin-resistant S. aureus (MRSA) strains that arise in hospitals as well as in the community (57). S. aureus USA300 is a highly virulent communityacquired MRSA strain that encodes many virulence factors,

\footnotetext{
${ }^{1}$ Institute for Biology-Microbiology, Freie Universität Berlin, Berlin, Germany.

${ }^{2}$ Center for Biotechnology, Bielefeld University, Bielefeld, Germany.

${ }^{3}$ Centre for Infection Medicine, Institute of Microbiology and Epizootics, Freie Universität Berlin, Berlin, Germany.

${ }^{4}$ Institute for Microbiology, Ernst-Moritz-Arndt-University of Greifswald, Greifswald, Germany.

${ }^{5}$ Laboratory of Structural Biochemistry and ${ }^{6}$ Institute of Chemistry and Biochemistry, Freie Universität Berlin, Berlin, Germany.
}

Vu Van Loi et al., 2018; Published by Mary Ann Liebert, Inc. This Open Access article is distributed under the terms of the Creative Commons Attribution Noncommercial License (http://creativecommons.org/licenses/by-nc/4.0), which permits and noncommercial use, distribution, and reproduction in any medium, provided the original authors and the source are cited. 


\section{Innovation}

Staphylococcus aureus is an important human pathogen that has to cope with the oxidative burst of the host innate immune system under infection conditions to ensure survival. Here, we have characterized the novel $\mathrm{NaOCl}-$ sensing transcription factor HypR that belongs to the Rrf2-family and controls the flavin disulfide reductase MerA under infection conditions. HypR senses and responds to $\mathrm{NaOCl}$ via the formation of Cys33-Cys99 intersubunit disulfides resulting in dissociation from its operator and derepression of hypR-merA transcription. The hypR-merA operon was required for growth and survival under $\mathrm{NaOCl}$ stress and in macrophage infection assays and was identified as an important defense mechanism under hypochlorite stress.

including the pore forming toxin Panton-Valentine leukocidin, the pyrogenic toxin superantigens (Sek and Seq), the plasminogen-activator staphylokinase (Sak), the staphylococcal complement inhibitor, and the chemotaxis inhibitory protein (Chp) contributing to the pathogenicity, immune evasion, and resistance to host defenses $(24,25,85)$. The pathogen is classified by the "European Center of Disease Prevention and Control" as ESKAPE pathogen because of its fast ability to escape the bactericidal action of antibiotics (76). Thus, to understand the adaptation of the pathogen to the host defense is of utmost importance to identify new drug targets for the treatment of MRSA infections.

During infections, $S$. aureus has to defend against reactive oxygen and chlorine species (ROS, RCS), such as hydrogen peroxide $\left(\mathrm{H}_{2} \mathrm{O}_{2}\right)$ and the strong oxidant hypochlorous acid $(\mathrm{HOCl})$ that are produced as first-line defense by activated macrophages and neutrophils with the aim to kill invading pathogens $(8,43,96,97)$. $\mathrm{HOCl}$ is generated in neutrophils from $\mathrm{H}_{2} \mathrm{O}_{2}$ and chloride by the myeloperoxidase (MPO), which is the main mechanism of bacterial killing $(39,48)$.

As ROS protection mechanism, $S$. aureus uses the lowmolecular-weight (LMW) thiol bacillithiol (BSH) that enhances survival in macrophage infection assays. In addition, various redox-sensing regulators of the SarA/MarR-family, such as MgrA and SarZ and the Staphylococcal accessory regulator (SarA), regulate ROS and antibiotic resistance determinants, as well as virulence factors (13-16, 43). MgrA controls the expression of several efflux pumps, which confer resistance toward fluoroquinolone, tetracycline, vancomycin, and penicillin. SarZ regulates the ohrA peroxiredoxin and genes involved in virulence, autolysis, cell wall biosynthesis, antibiotic resistance, and energy metabolism. The OhrA peroxiredoxin reduces organic hydroperoxides that are derived from fatty acid oxidation to their organic alcohols (26). Both SarZ and MgrA use a thiol-based redox-sensing mechanism in response to organic hydroperoxides $(78,88)$. The single Cys residue of SarZ and MgrA can be regulated by $S$-thiolation with a synthetic LMW thiol leading to allosteric changes in the DNA-binding domains and derepression of transcription of the target genes $(1,15,43)$.

To identify novel sodium hypochlorite $(\mathrm{NaOCl})$-sensitive proteins, we have recently applied the OxICAT method and studied the quantitative thiol-redox proteome of $S$. aureus USA300 under $\mathrm{NaOCl}$ stress (46). The redox state of 228 Cys residues in $S$. aureus could be quantified and $58 \mathrm{NaOCl}-$ sensitive Cys residues were identified with $>10 \%$ increased oxidations under $\mathrm{NaOCl}$ treatment. Of note, the redoxsensing Cys residues of both MarR/OhrR-family regulators MgrA and SarZ showed increased oxidation levels under $\mathrm{NaOCl}$ stress. The $\mathrm{NaOCl}$-sensitive proteins include also many antioxidant proteins, detoxification and metabolic enzymes, ribosomal proteins, several $\mathrm{Zn}$-containing proteins, and virulence factors. Moreover, five $S$-bacillithiolated proteins were identified under $\mathrm{NaOCl}$ stress, including Gap, AldA, GuaB, and RpmJ that showed the highest oxidation increase of $>20 \%$ in the OxICAT analysis. Gap contributes as most abundant $S$-bacillithiolated protein in the proteome with $4 \%$ to the total Cys proteome of $S$. aureus. We have further shown that $S$-bacillithiolation of Gap functions in redox control and thiol protection under $\mathrm{H}_{2} \mathrm{O}_{2}$ and $\mathrm{NaOCl}$ stress in vitro (46). However, while our redox proteomics studies identified many new targets for redox regulation, no $\mathrm{HOCl}$-specific transcription factor was identified that could be specifically involved in sensing of $\mathrm{NaOCl}$ stress.

In this study, we applied RNA-seq transcriptomics to identify new redox-sensing regulators in S. aureus USA300 that could specifically sense and respond to $\mathrm{NaOCl}$ stress and thereby regulate target genes that confer protection under infection conditions. The hypR-merA operon was most strongly upregulated under $\mathrm{NaOCl}$ in the transcriptome that encodes for the novel redox-sensing regulator HypR and the pyridine nucleotide disulfide reductase mercuric ion reductase (MerA). HypR belongs to the widespread Rrf2 family of transcriptional regulators that include iron/sulfur (FeS)-cluster redox sensors for nitric oxide (NsrR) $(23,90,98)$, the iron/ sulfur status of the cell (IscR) (82, 83), iron metabolism (RirA) (40), or other signals (67). Other Rrf2 family regulators do not contain FeS clusters, such as the cysteine metabolism repressor (CymR) $(28,47,84)$ and the redox-sensing SaiR repressor of Bacillus anthracis that controls spxA2 under disulfide stress conditions and is a close homologue of HypR (68). Using biochemical and genetic approaches, we have characterized HypR as a novel thiol-based redox sensor that senses $\mathrm{HOCl}$ stress by intersubunit disulfide formation and protects $S$. aureus under macrophage infection conditions.

\section{Results}

$\mathrm{NaOCl}$ stress causes an oxidative stress response in the transcriptome and upregulates most strongly the hypR-merA operon (USA300HOU_0588-87)

in $\mathrm{S}$. aureus USA300

We were interested to identify novel redox-sensing regulators in $S$. aureus that could be specifically involved in the hypochlorite defense under infection conditions. Thus, we studied the changes in the RNA-seq transcriptome in $S$. $a u$ reus USA300 after exposure to sublethal $150 \mu M \mathrm{NaOCl}$ stress compared to untreated control cells. The $\mathrm{NaOCl}$ stress experiments were performed in Belitsky minimal medium (BMM) to avoid quenching by rich medium components as described in our previous studies $(46,58)$. Three biological replicate experiments were performed and the samples for RNA isolation were taken before (control) and $30 \mathrm{~min}$ after $\mathrm{NaOCl}$ stress and subjected to RNA-seq analysis as described (44). For significant expression changes, the $\log 2$ fold change (m-value) cutoff ( $\log 2$-fold change $\mathrm{NaOCl} v s$. control) 
of \pm 1.98 was chosen ( $95 \%$ confidence, $p \leq 0.05$ ). In total, 89 transcripts were significantly at least fourfold upregulated and 9 were fourfold downregulated under $\mathrm{NaOCl}$ stress in the transcriptome data set (Fig. 1 and Supplementary Table S1; Supplementary Data are available online at www.liebertpub. com/ars). In addition, 1442 genes are differentially transcribed below the m-value cutoff under $\mathrm{NaOCl}$ stress in the RNA-seq transcriptome of $S$. aureus USA300. All induced and repressed genes above and below the m-value cutoff were sorted into regulons, and a subset of the most interesting upregulated regulons is displayed in Voronoi treemaps (Fig. 2 and Supplementary Table S2).

Previous transcriptome analysis in Bacillus subtilis showed that $\mathrm{NaOCl}$ stress induces an oxidative stress response as revealed by the induction of the oxidative stress-specific PerR, OhrR, and Spx regulons, as well as by the CtsR regulon indicative of oxidative protein unfolding (20). Furthermore, $\mathrm{NaOCl}$ stress resulted in increased expression of the YodB, CatR, HypR, and HxlR regulons in B. subtilis that sense reactive electrophilic species (RES), such as quinones and aldehydes $(18,20,21,52,73)$. In $S$. aureus, we could confirm a similar expression profile under $\mathrm{NaOCl}$ stress as revealed by the strong upregulation of the PerR, HrcA, and QsrR (YodB) regulons indicating ROS and RES responses in $S$. aureus. In addition, we noticed the weak two- to threefold induction of few members of the Fur and Zur regulons. Fur and Zur oxidation in their Zn-redox switch motifs was observed under $\mathrm{NaOCl}$ stress in our recent OxICAT analyses in S. aureus (46) and Mycobacterium smegmatis (44). Similarly, the Fur family protein PerR was oxidized to an intramolecular disulfide in B. subtilis under $\mathrm{NaOCl}$ stress (20). Since Zn-redox switch motifs are known sensors for $\mathrm{HOCl}$ stress also in $E$. coli (45, 95), redox regulation of PerR, Fur, and Zur could involve also thiol oxidation in their Zn-motifs.

The SigmaB regulon was also induced under $\mathrm{NaOCl}$ stress in $S$. aureus and B. subtilis, which might be indicative for an acid stress response and contributes to virulence (20) (Fig. 2). The induction of the SigmaB regulon was previously shown after internalization of $S$. aureus in human epithelial cells and SigmaB was essential for intracellular growth (77). Of note is further induction of GraRS cell wall stress regulon under $\mathrm{NaOCl}$ stress in $\mathrm{S}$. aureus. GraRS controls resistance to antimicrobial peptides and cell wall-active antibiotics, such as vancomycin, but contributes also to the oxidative stress resistance of $S$. aureus explaining its upregulation under $\mathrm{NaOCl}$ stress (29). The oxidative stress response is further indicated by induction of genes that encode for BSH biosynthesis enzymes and bacilliredoxins, such as $b \operatorname{sh} A, b \operatorname{sh} B, b \operatorname{sh} C$, and $\operatorname{brx} B$ in $S$. aureus. The BSH biosynthesis genes are under control of Spx in B. subtilis (33) and perhaps also in S. aureus.

Apart from the stress regulons, the SaeRS regulon was most strongly induced under $\mathrm{NaOCl}$ stress in S. aureus. The SaeRS two-component system controls many important toxins, such as $\alpha-, \beta$-, and $\gamma$-hemolysins, leukocidins, superantigens, surface proteins, proteases, nuclease, coagulase, and immune evasion proteins $(7,38,56)$ that exhibit the highest fold change between 20- and 160-fold under $\mathrm{NaOCl}$ stress (Supplementary Tables S1 and S2, Figs. 1 and 2). Previous studies established important roles of SaeRS in pathogenicity and biofilm formation in a variety of disease models of infections (56). The SaeRS regulon was most strongly upregulated by neutrophil stress in S. aureus as shown in previous microarrays (91). The

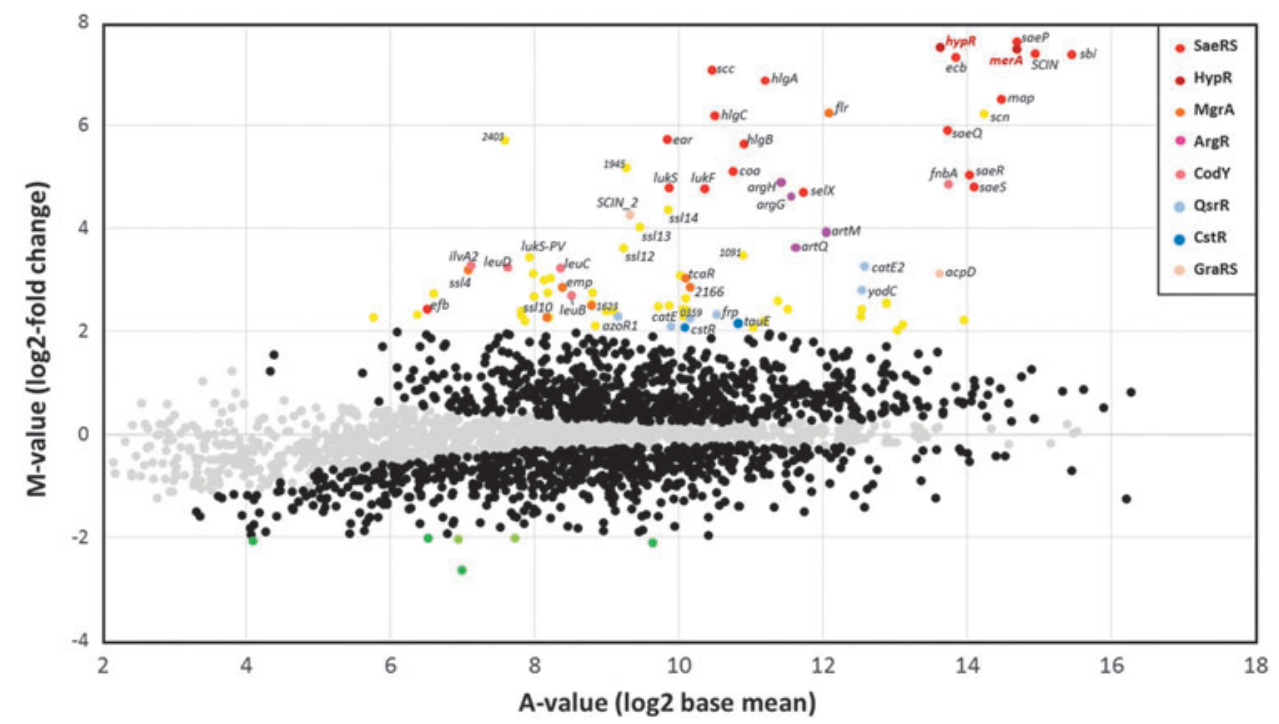

FIG. 1. RNA-seq transcriptomics of Staphylococcus aureus USA300 after 30 min of NaOCl stress. For RNA-seq transcriptome profiling, $S$. aureus USA300 was grown in BMM and treated with $150 \mu \mathrm{M} \mathrm{NaOCl}$ stress for 30 min. The gene expression profile under $\mathrm{NaOCl}$ stress is shown as ratio/intensity scatter plot (M/A-plot), which is based on the differential gene expression analysis using DeSeq2. Colored symbols indicate significantly induced (red, magenta, yellow) or repressed (green) transcripts (M-value $\geq 1.98$ or $\leq-1.98$; $p$-value $\leq 0.05$ ). Black symbols indicate differential transcribed genes below the M-value cutoff of $1.98>\mathrm{M}>-1.98(p \leq 0.05)$. Gray symbols denote transcripts with no fold changes after $\mathrm{NaOCl}$ stress $(p>0.05)$. The SaeRS, HypR, MgrA, ArgR, CodY, and QsrR regulons are most strongly upregulated under NaOCl stress. The transcriptome analysis was performed from three biological replicates. The RNA-seq expression data of all genes after $\mathrm{NaOCl}$ stress and their regulon classifications are listed in Supplementary Tables S1 and S2. BMM, Belitsky minimal medium; $\mathrm{NaOCl}$, sodium hypochlorite. 


\section{S. aureus $\mathrm{NaOCI}$ RNA-seq transcriptome}

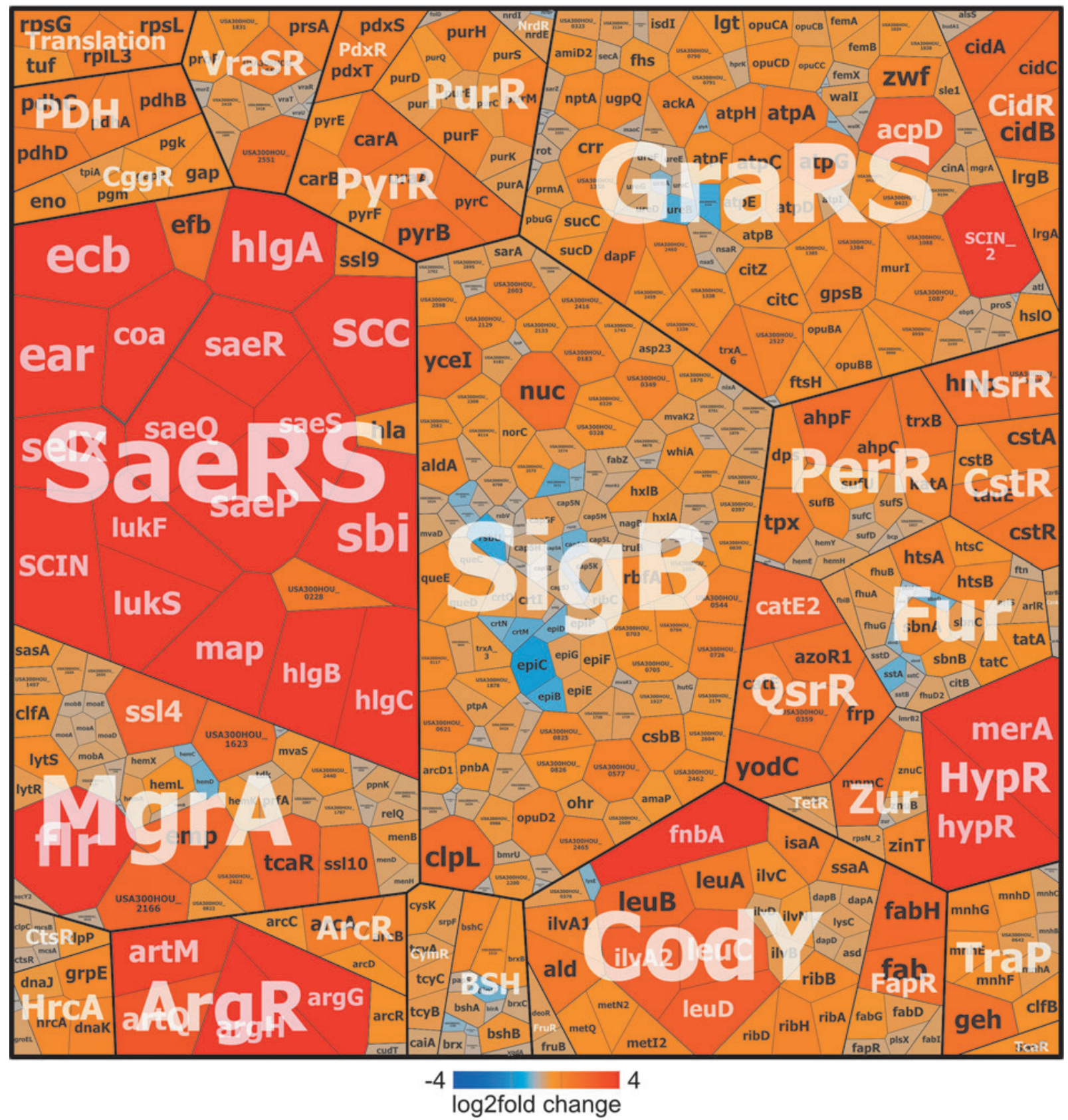

FIG. 2. The transcriptome treemap of $S$. aureus USA300 under NaOCI stress indicates an oxidative stress response and the strong upregulation of the HypR and SaeRS regulons. The transcriptome treemap shows the differential gene expression of $S$. aureus after exposure to $150 \mu \mathrm{M} \mathrm{NaOCl}$ stress as $\log 2$ fold changes (m-values). The genes are classified into operons and regulons based on the RegPrecise database and previous publications (61). Differential gene expression is visualized using a red-blue color code where red indicates $\log 2$ fold induction and blue repression of transcription under $\mathrm{NaOCl}$ stress. The HypR and SaeRS regulons are most strongly upregulated under $\mathrm{NaOCl}$ stress in S. aureus USA300. The induction of the PerR, HrcA, SigmaB, and QsrR regulons reveals an oxidative stress response in S. aureus. The RNA-seq expression data of the selected highly transcribed genes after $\mathrm{NaOCl}$ stress and their regulon classifications are listed in Supplementary Table S2.

SaeRS-controlled virulence factors are essential for innate immune evasion since the survival of the saeRS mutant was impaired in neutrophil phagocytosis assays (92). Reduction of ROS production by SaeRS-regulated virulence factors inside neutrophils was recently shown as mechanism to avoid pathogen killing (37). Thus, the strong induction of the SaeRS virulence regulon under $\mathrm{NaOCl}$ stress in our transcriptome is reflecting adaptation to infection conditions, such as neutrophil stress.

Among the genes involved in energy metabolism, the expression of the glycolytic cggR-gap-pgk-tpiA-pgm-eno-operon and the pyruvate dehydrogenase $p d h A B C D$ operon was two- to 
fourfold elevated. Since the glycolytic Gap is the main thiol switch that is redox controlled and inhibited under $\mathrm{NaOCl}$ stress by $S$-bacillithiolation (46), the lack of energy production due to inhibited glycolysis could result in upregulation of the glycolysis enzymes. Furthermore, the two- to threefold induction of the ATP synthase atpCDGAHFEB operon under $\mathrm{NaOCl}$ stress may indicate ATP depletion. Of note is the further enhanced expression of the $\mathrm{CodY}$ regulon under $\mathrm{NaOCl}$ that includes the $i l v$-leu and lysC-asd-dapABD operons involved in leucine, isoleucine, and lysine biosynthesis, as well as the riboflavin biosynthesis ribHBAED operon. Increased demand of riboflavin might be caused by the requirement of flavin cofactors for thiol/disulfide reductases. Moreover, the ArgR regulon genes for biosynthesis of arginine and the ArcRcontrolled arginine deiminase operon are strongly induced under $\mathrm{NaOCl}$, which could indicate increased ammonium production to compensate for an acid stress response caused by $\mathrm{NaOCl}$ stress.

Most interesting was the 180-fold induction of the USA300HOU_0588-0587 operon under $\mathrm{NaOCl}$ treatment (Figs. 2 and 3). USA30OHOU_0588 encodes for an uncharacterized Rrf2 family regulator, termed as HypR, and USA300HOU_0587 encodes for the flavin disulfide reductase (FDR) MerA. The strong expression of the hypR-merA operon was previously observed in $S$. aureus isolates during phagocytosis with polymorphonuclear leukocytes (PMNs or neutrophils) (91). Thus, our expression profile under $\mathrm{NaOCl}$ stress highly resembles that of infection conditions due to the production of ROS and RCS in activated neutrophils. Accordingly, HypR could specify a novel $\mathrm{NaOCl}-$ specific transcription factor and defense mechanism of $S$. aureus to avoid destruction by neutrophils. Thus, we studied the redox-sensing regulatory mechanism of HypR and the function of MerA in more detail under $\mathrm{NaOCl}$ stress conditions. For the detailed HypR functional analysis, we have chosen $S$. aureus COL, which is our model strain for functional genomics, and protocols for genetic manipulation are best established in our laboratory for COL, which has less resistance determinants compared to USA300.

\section{Northern blot analysis revealed the specific induction of the hypR-merA operon under $\mathrm{NaOCl}$ and diamide stress and its negative regulation by the Rrf2 family repressor HypR}

We used Northern blot analysis to study the expression of the hypR-merA operon under different thiol-specific stress conditions in $S$. aureus $\mathrm{COL}$, including $1 \mathrm{~m} M \mathrm{NaOCl}, 2 \mathrm{~m} M$ diamide, $10 \mathrm{mM} \mathrm{H} \mathrm{O}_{2}, 0.75 \mathrm{~m} M$ formaldehyde, and $0.5 \mathrm{~m} M$ methylglyoxal (Fig. 4A). These experiments were performed in RPMI medium, which resembles infection conditions and allows faster growth of $S$. aureus, but requires higher sublethal doses of $1 \mathrm{~m} M \mathrm{NaOCl}$. The strong upregulation of the hypR-merA-specific $1.8 \mathrm{~kb}$ mRNA under $1 \mathrm{~m} M \mathrm{NaOCl}$ and $2 \mathrm{~m} M$ diamide stress was confirmed, but no significant in-

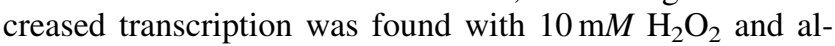
dehyde stress. This indicates that the hypR-merA operon responds specifically to disulfide stress, but not to $\mathrm{H}_{2} \mathrm{O}_{2}$ and aldehydes in $S$. aureus.

Next, we studied the role of HypR in transcriptional regulation of the hypR-merA operon in $S$. aureus COL. Using the temperature-sensitive plasmid pMAD, a clean hypR deletion mutant was constructed lacking $364 \mathrm{bp}$ of the hypR open reading frame. The truncated hypR-merA transcript of $1.5 \mathrm{~kb}$ (denoted as TR-merA) was constitutively expressed in the hyp $R$ deletion mutant under nonstress conditions, indicating full derepression of hypR-merA transcription (Fig. 4B). These results identify HypR as transcriptional repressor of the hypR-merA operon that is inactivated under disulfide stress conditions in $S$. aureus.
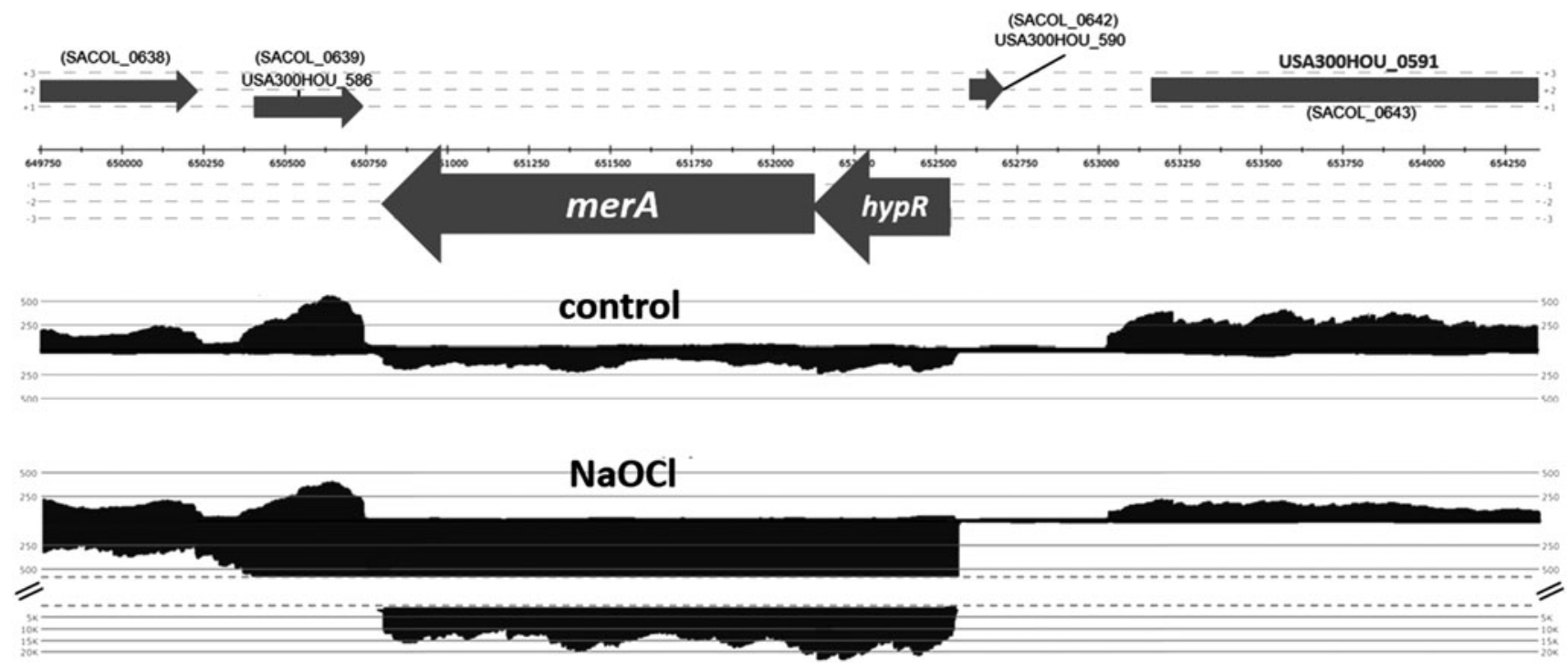

FIG. 3. The hypR-merA-operon is most strongly upregulated in the RNA-seq transcriptome of $S$. aureus USA300 under NaOCl stress. The mapped reads for the gene expression profile of the hypR-merA locus under control and $\mathrm{NaOCl}$ stress are shown as displayed using the Read-Explorer software. Transcription of the hypR-merA-operon is 180 -fold induced under $\mathrm{NaOCl}$ stress in $S$. aureus USA300. The hypR gene encodes for an Rrf 2 transcriptional regulator and merA encodes for a pyridine nucleotide disulfide reductase. 


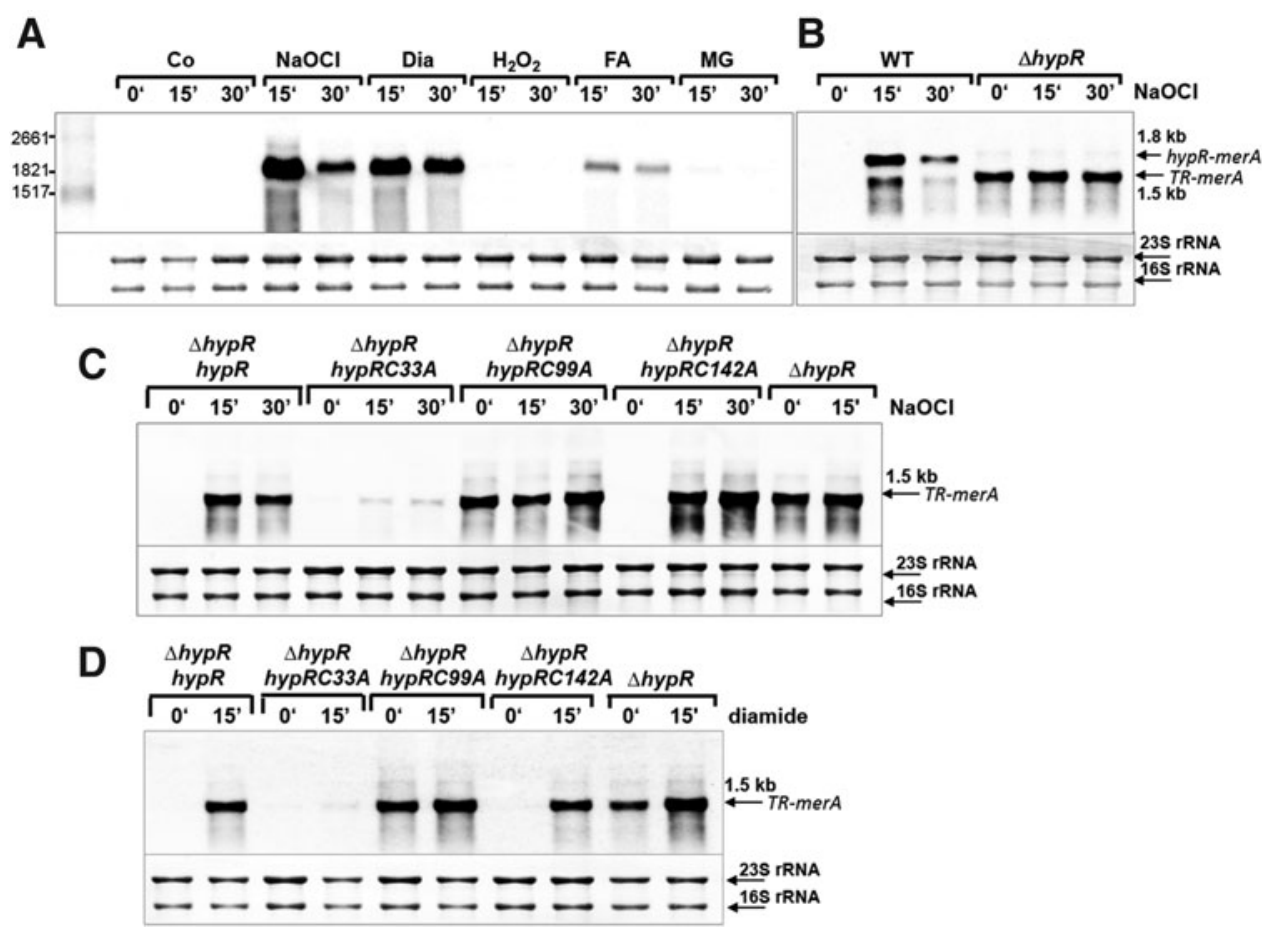

FIG. 4. Northern blot analysis of hypR-merA transcription in $\mathrm{S}$. aureus $\mathrm{COL}$ under $\mathrm{NaOCl}$, diamide, $\mathrm{H}_{2} \mathrm{O}_{2}$, and aldehyde stress and in hypR Cys-Ala mutants. (A) Northern blot analysis was performed using RNA isolated from $S$. aureus COL wild type before (co) and 15 and $30 \mathrm{~min}$ after exposure to $1 \mathrm{~m} M \mathrm{NaOCl}, 2 \mathrm{~m} M$ diamide, $10 \mathrm{~m} M \mathrm{H}_{2} \mathrm{O}_{2}, 0.75 \mathrm{~m} M$ formaldehyde, and $0.5 \mathrm{~m} M$ methylglyoxal stress. (B) Transcription of the hypR-merA operon was analyzed in the COL wildtype and in the $\Delta h y p R$ mutant under $1 \mathrm{~m} M \mathrm{NaOCl}$ stress indicating strong derepression of hypR-merA transcription under control conditions in the absence of HypR. (C, D) Northern blot analysis of hypR-merA operon transcription in the $\Delta h y p R$ deletion mutant and in $\triangle h y p R$ mutants complemented with hypR, hypRC33A, hypRC99A, and hypRC142A before and 15 and $30 \mathrm{~min}$ after exposure to $1 \mathrm{mM} \mathrm{NaOCl}(\mathbf{C})$ or $2 \mathrm{~m} M$ diamide stress (D). The results indicate that Cys 33 is required for redox sensing of HypR in vivo. For stress experiments, $S$. aureus cells were grown in RPMI medium and treated with thiolreactive compounds at an $\mathrm{OD}_{500}$ of 0.5 . The arrows point toward the hypR-merA bicistronic mRNA (1.8 kb) in the wild type or the truncated hypR-merA transcript (TR-merA) $(1.5 \mathrm{~kb})$ in the hypR mutant. The methylene blue stain is the RNA loading control indicating the $16 \mathrm{~S}$ and $23 \mathrm{~S}$ rRNAs. $\mathrm{OD}_{500}$, optical density at $500 \mathrm{nms} . \mathrm{H}_{2} \mathrm{O}_{2}$, hydrogen peroxide.

Cys99 is required for HypR repressor activity and Cys33 and Cys99 are the $\mathrm{NaOCl}$-sensing Cys residues in vivo

The HypR repressor belongs to the Rrf2 family that includes FeS cluster containing redox sensors for nitric oxide (NsrR) $(23,90,98)$, and also SaiR of $B$. anthracis as the closest HypR homologue that does not contain an FeS cluster (68) (Fig. 5A). Three conserved Cys residues (Cys93, Cys99, and Cys 105) and Asp8 are essential for FeS cluster coordination in NsrR (90), but only Cys99 is conserved in HypR and SaiR. Instead, HypR has two other Cys residues at the nonconserved positions 33 and 142. The crystal structure of the HypR and SaiR homologue YwnA of B. subtilis is available (PDB code 1xd7), which showed 23.5\% sequence identity to HypR and shares also the conserved Cys97 (Fig. 5B). Based on the structure of YwnA as template, the HypR and SaiR structures were modeled with SWISSMODEL (10). The HypR structural model indicates that Cys33 is located in the turn between the $\alpha 2$ and $\alpha 3$ DNA recognition helices, while Cys99 is at the N-terminus of the long $\alpha 6$ helix that forms the dimer interface (Fig. 5B). The position of Cys 142 could not be modeled since the Cterminal $\alpha 8$ helix is not present in the YwnA structure that was used as template.
To study the role of the three Cys residues for HypR repressor activity and redox sensing, we analyzed transcription of the hypR-merA operon in hypRC33A, hypRC99A, and hypRC142A mutants in vivo. The successful complementation of the hypR mutant using the pRB473-based expression of hypR, hypRC33A, hypRC99A, and hypRC142A under control, $\mathrm{NaOCl}$ and diamide stress was confirmed using Northern blots with a hypR-specific mRNA probe (Supplementary Fig. S1A, B). Additional Western blot analysis confirmed that the complemented strains expressed HypR, Cys33A, Cys99A, and Cys142A mutant proteins at the same protein levels (Supplementary Fig. S6A, B). Transcriptional analysis of hypR$m e r A$ expression revealed that the hypRC33A mutation did not affect the DNA binding activity of HypR, but impaired its redox sensing since transcription was abolished under $\mathrm{NaOCl}$ and diamide stress (Fig. 4C, D). In contrast, the hypRC99A mutant was impaired in DNA binding as shown by full derepression of the hypR-merA operon under control conditions. The hypRC142A mutant behaved similar as the wild type and was not impaired in DNA binding and redox sensing under $\mathrm{NaOCl}$ and diamide stress. In conclusion, our Northern blot results revealed that Cys33 is important for redox sensing under $\mathrm{NaOCl}$ and diamide stress and $\mathrm{Cys} 99$ is required for HypR repressor activity in vivo. 

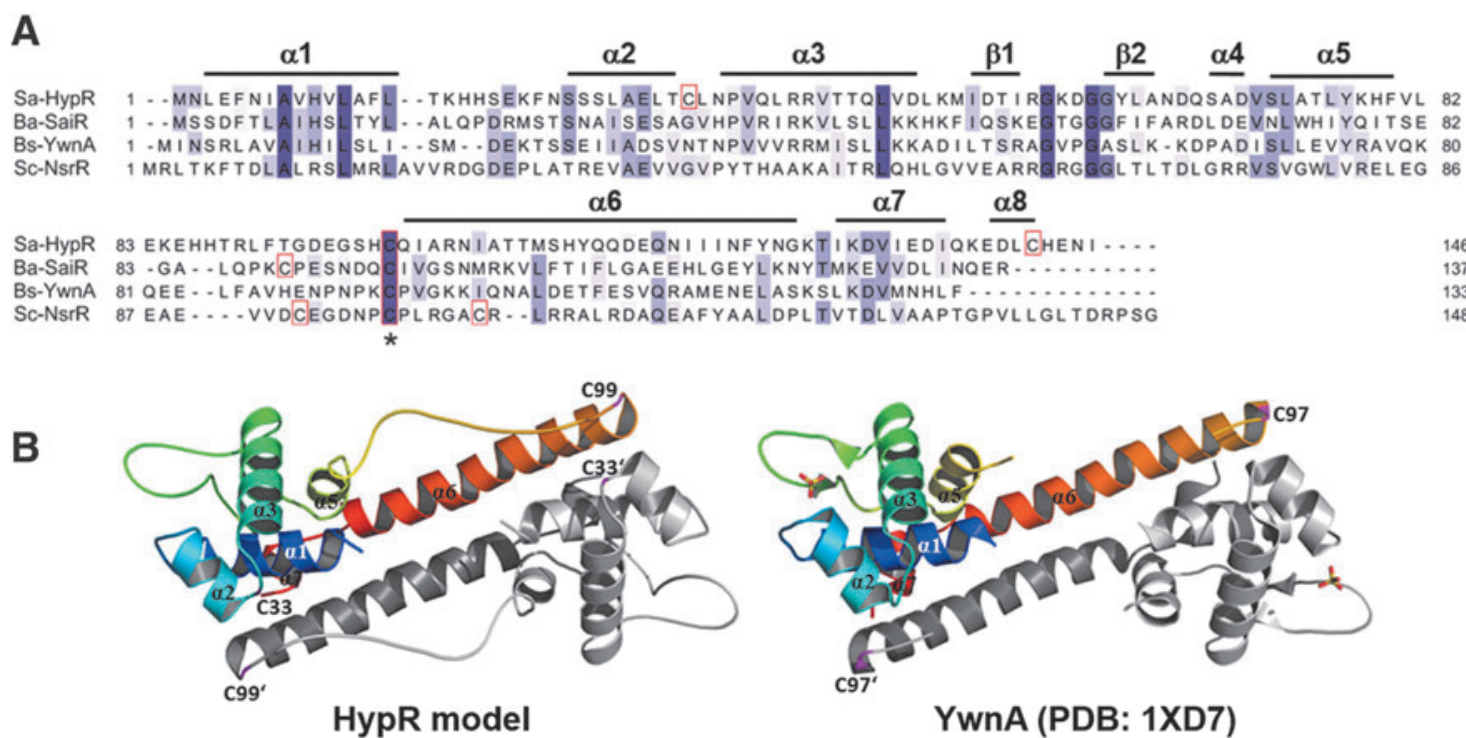

YwnA (PDB: 1XD7)

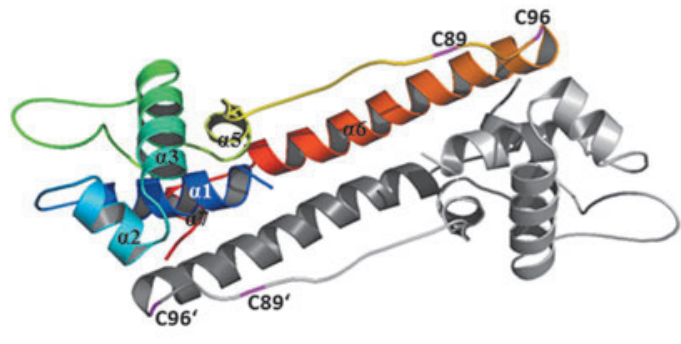

SaiR model

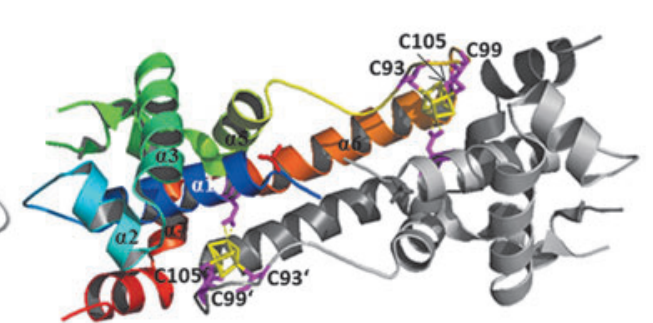

NsrR (PDB: 5N07)

FIG. 5. Multiple protein sequence alignments of the Rrf2 regulators HypR, SaiR, YwnA, and NsrR (A) and structural modeling of HypR and SaiR in comparison to YwnA and NsrR (B). (A) The protein sequence alignment was performed with Clustal $\Omega 2$ and is presented in Jalview. The following protein sequences were aligned and the $\%$ identity to HypR is given in parenthesis: HypR (SACOL0641) of S. aureus COL, SaiR (BAS3200) of Bacillus anthracis (20.4\%), YwnA (P71036) of Bacillus subtilis (23.48\%), and NsrR (Q9L132) of Streptomyces coelicolor (17.86\%). Intensity of the blue color gradient is based on 50\% sequence identity. The conserved Cys99 in HypR is labeled in red with an asterisk (*). (B) The structural models of HypR and SaiR were generated using SWISS-MODEL (10) and visualized with PyMOL using the template of Bacillus subtilis YwnA (1xd7) that showed $23.5 \%$ and $25.78 \%$ sequence identity to HypR and SaiR, respectively. For comparison, we show the structures of YwnA (1xd7) and NsrR (5no7) with labels for the conserved Cys97 in YwnA and the 3 FeS cluster coordinating Cys residues (Cys93, Cys99, and Cys105) in NsrR. The FeS cluster of NsrR is displayed in yellow. FeS, iron/sulfur; NsrR, redox sensors for nitric oxide.

HypR binds specifically to the hypR-merA operator and DNA binding is reversibly inhibited under $\mathrm{NaOCl}$ and diamide stress in vitro

The results above suggested that HypR binds directly to the hypR-merA promoter region and that Cys33 is the redoxsensing Cys residue that is oxidized under $\mathrm{NaOCl}$ and diamide stress. Rrf 2 regulators are known to bind as homodimers to operator sequences with dyad symmetry (84). Based on the RNA-seq data, we used the MEME software to identify the putative promoter sequence and a conserved inverted repeat in the upstream region of the hypR-merA operon (Fig. 6A). The 12-3-12 bp inverted repeat sequence TAATTGTAACTA-N $3^{-}$ CAGTTACAATTA was detected in the hypR-merA upstream region as possible HypR binding site that overlapped with the putative -10 region. Similar inverted repeats are characteristic as full-length binding sites for other Rrf2 regulators, such as SaiR, CymR, IscR, and RsrR $(67,68,82,84)$. We searched for the conservation of the putative HypR operator sequence up- stream of homologous hypR-merA operons in the genomes of other Staphylococcus species. The multiple-sequence alignment showed that the $12-3-12$ bp inverted repeat sequence is highly conserved in the hypR-merA upstream promoter regions across Staphylococci (Fig. 6B).

To investigate whether HypR binds directly and specifically to the HypR operator sequence under reduced conditions with dithiothreitol (DTT), we used electrophoretic mobility shift assays (EMSAs). The gel shift results revealed that purified reduced HypR protein binds with high affinity to the hypRmerA upstream region in vitro (Fig. 7A). To analyze the specific binding of HypR to the 12-3-12 inverted repeat sequence, we exchanged two nucleotides in each half of the inverted repeat by G-T and A-C (IR-m1 probe) or T-G and C-A (IR-m2 probe) and analyzed the DNA-binding activity of HypR to these mutated DNA probes (Supplementary Fig. S2). HypR was unable to bind to the mutated inverted repeats IR-m1 and IR-m2 in vitro supporting the specific binding of HypR to the identified operator sequence. Furthermore, HypR was also 
A
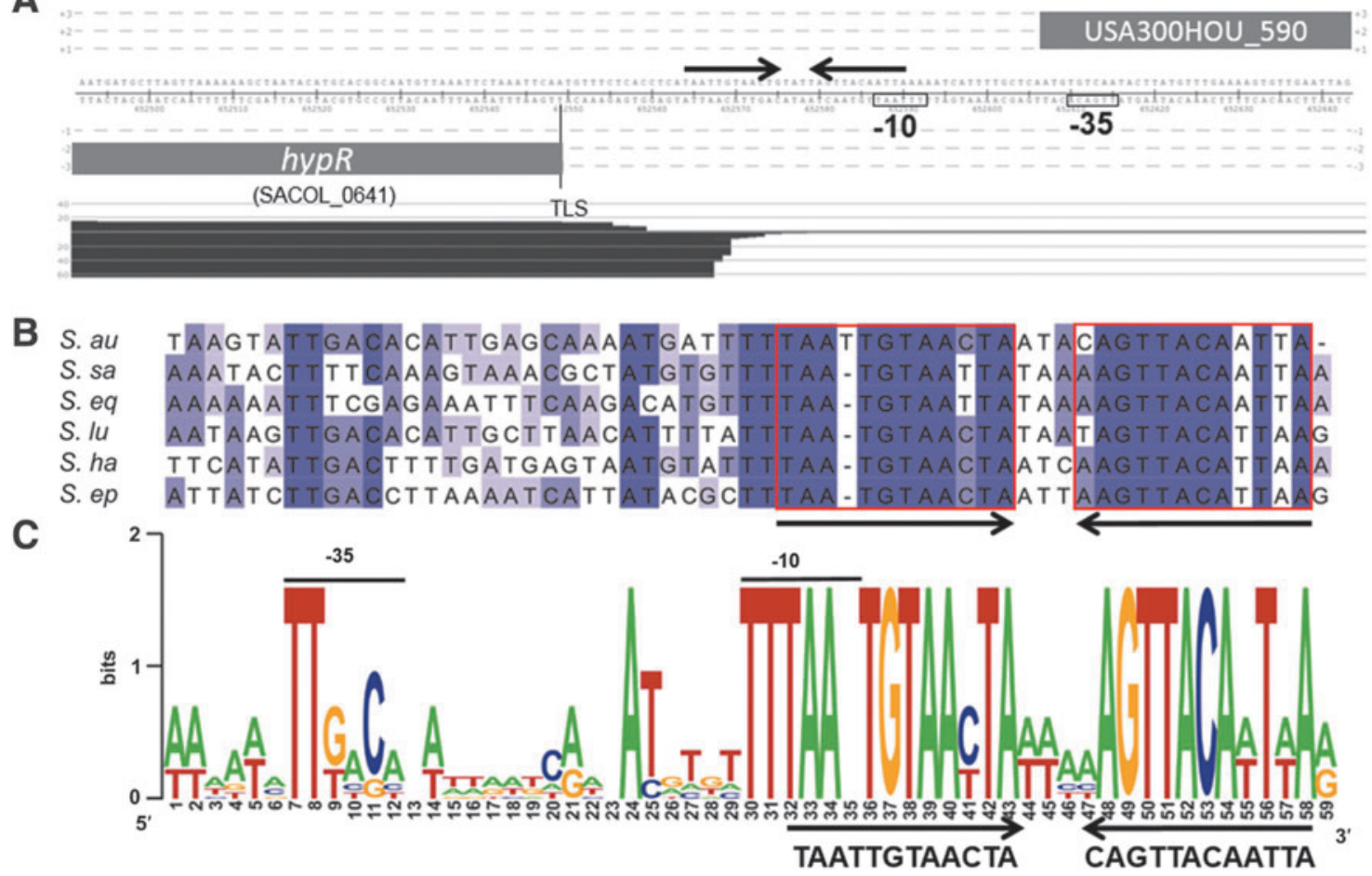

FIG. 6. Alignment of the hypR-merA upstream promoter regions with the 12-3-12 bp inverted repeat in Staphylococcus species. The upstream promoter region of the hypR-merA operon includes a 12-3-12 bp inverted repeat sequence that is highly conserved upstream of other hypR-merA homologues across Staphylococci, including S. aureus (SACOLO641), Staphylococcus saprophyticus (SSP2349), Staphylococcus equorum (SE1039_01590), Staphylococcus lugdunensis (SLGD_02231), Staphylococcus haemolyticus (SH2331), and Staphylococcus epidermidis (SE0366). (A) Upper panel shows the reads mapped for the hypR-merA transcript as visualized using ReadExplorer. The putative -10 and -35 promoter sequences are labeled and the 12-3-12 bp conserved inverted repeat is boxed and indicated by arrows. (B) All upstream promoter sequences of hypR-merA homologues were aligned using Clustal $\Omega 2$ and presented in Jalview. Intensity of the blue color gradient is based on 50\% nucleotide sequence identity. (C) Bottom panel represents the 12-3-12 bp conserved inverted repeat created with WebLogo as HypR operator sequence.

unable to bind to nonspecific DNA probes (trxA and RNAIII genes) (Supplementary Fig. S2B).

Next, the effects of Cys-Ala mutations in Cys33, Cys99, and Cys142 on the DNA-binding activity of HypR were investigated using gel shift experiments. The reduced HypRC33A and HypRC142A mutant proteins showed similar DNA-binding affinities compared with the HypR wild-type protein (Fig. 7A). However, the reduced HypRC99A and HypRC99S mutant proteins were unable to bind to the hypRmerA promoter, which confirms our in vivo results. Next, we analyzed the effect of thiol oxidation of the HypR, HypRC33A, and HypRC142A proteins on DNA-binding activity in vitro. Treatment of HypR, HypRC33A, and HypRC142A mutant proteins with $1-20 \mu M \mathrm{NaOCl}$ or $10-20 \mu M$ diamide resulted in their fast dissociation from the hypR operator DNA (Fig. 7B, C). This indicates that HypR as well as the HypRC33A and HypRC142A mutant proteins respond similar to $\mathrm{NaOCl}$ and diamide stress in vitro. The reduction of the oxidized HypR proteins with DTT restored the DNA-binding ability indicating that $\mathrm{HypR}$ responds to $\mathrm{NaOCl}$ stress by reversible thiol oxidation. The responsiveness of the HypRC33A mutant to $\mathrm{NaOCl}$ stress in vitro is in contrast to lack of $\mathrm{NaOCl}$ response and hypR-merA transcription in the hypR$C 33 A$ mutant in vivo. Cys33 was identified as redox-sensing
Cys in vivo, but the C33A mutant was not impaired in redox sensing under $\mathrm{NaOCl}$ and diamide in vitro. It might be possible that the purified C33A mutant protein is oxidized to the "wrong" disulfides under disulfide stress in vitro, which are not stabilized inside $S$. aureus. In summary, our results show that HypR is a redox-sensing regulator that controls expression of the hypR-merA operon and is inactivated due to reversible thiol oxidation under $\mathrm{NaOCl}$ and diamide stress leading to release from the promoter DNA.

\section{HypR responds to $\mathrm{NaOCl}$ and diamide stress by intermolecular disulfide formation that involves the redox-sensing Cys33 and Cys99}

The EMSA results and Northern blots revealed that the DNA-binding activity of HypR is redox regulated by reversible thiol oxidation under $\mathrm{NaOCl}$ and diamide stress. Thus, we were interested to elucidate the thiol switch mechanism leading to release of HypR from the target DNA. HypR can be classified as 2-Cys-type redox regulator, such as OhrR from Xanthomonas campestris and YodB from B. subtilis that sense different thiol reactive compounds by intermolecular disulfide formation between the opposing subunits in the homodimer (2, 27, 43, 74). Thus, HypR most likely also senses and responds 


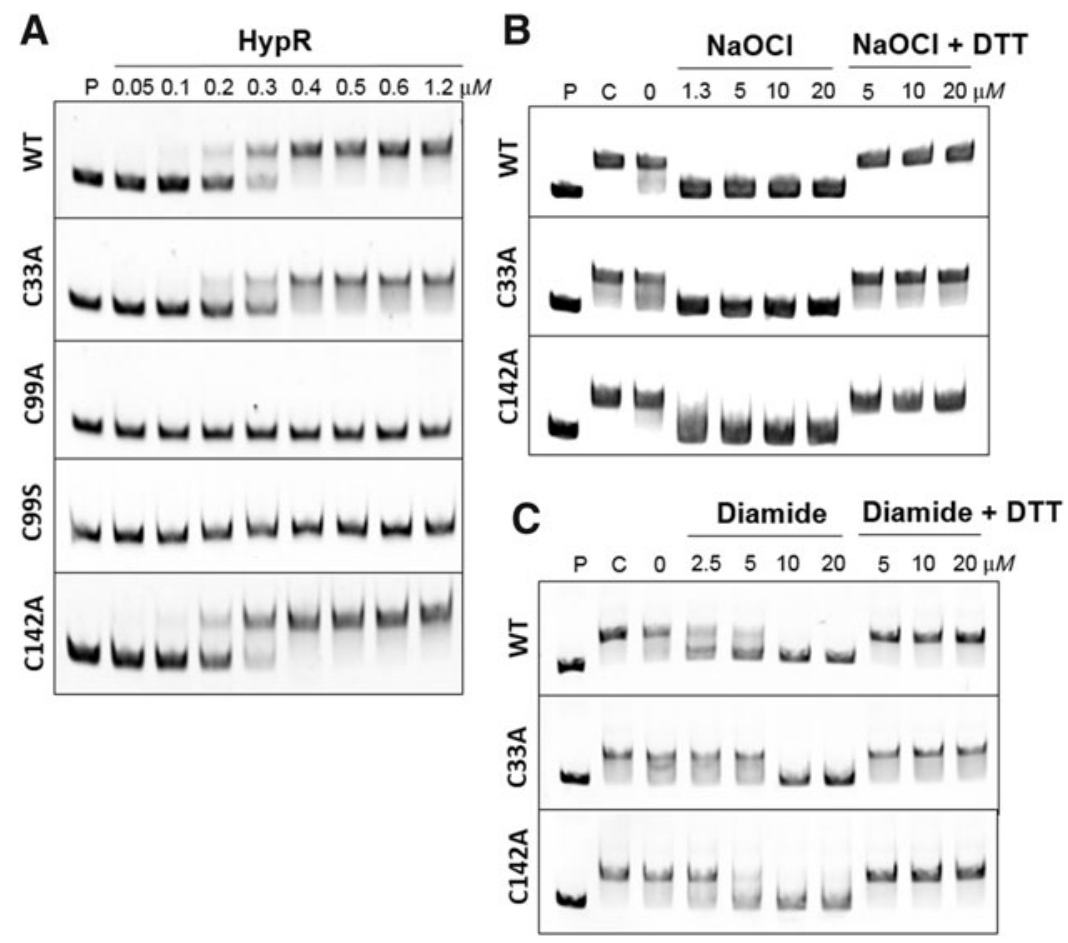

FIG. 7. DNA binding of HypR is inhibited by reversible thiol oxidation under $\mathrm{NaOCl}$ and diamide stress in vitro and the effect of HypR Cys mutations on DNA binding and redox sensing. (A) EMSAs were used to analyze the DNAbinding activity of purified HypR, HypRC33A, HypRC99A, and HypRC142A proteins to the hypR-merA upstream promoter region in vitro. Increasing concentrations $(0.05-1.25 \mu M)$ of HypR were used in the DNA-binding reactions with $0.75 \mathrm{ng}$ of template DNA ranging from -128 to +70 relative to the transcription start site of the hypR-merA operon. $(\mathbf{B}, \mathbf{C})$ DNA-binding activity of HypR, HypRC33A, and HypRC142A proteins was inhibited by $1.3-20 \mu \mathrm{M} \mathrm{NaOCl}$ (B) or 10$20 \mu M$ diamide (C) and could be restored with $1 \mathrm{~m} M$ DTT. This indicates that HypR resembles a redox-sensing regulator that is inactivated due to reversible thiol oxidation. The HypRC99A and HypRC99S mutants were unable to bind to the hypR-merA target promoter. "P" indicates the free probe, "C" is the HypR-DNA complex in the presence of DTT, and " 0 ", indicates the control of HypR-DNA complex after DTT removal before exposure to NaOCl. DTT, dithiothreitol; EMSA, electrophoretic mobility shift assay.

to $\mathrm{NaOCl}$ via intersubunit disulfide bonds. Indeed, when we exposed purified HypR to $\mathrm{NaOCl}$ or diamide stress, it was quickly oxidized to the disulfide-linked dimer that migrates at the size of $35 \mathrm{kDa}$ in the nonreducing SDS-PAGE (Fig. 8A and Supplementary Fig. S4A). The oxidation of HypR to the intersubunit disulfide was reversible with DTT as shown in the reducing SDS-PAGE (Supplementary Figs. S3A and S4C). Of note, two bands migrated closely together at the size of the HypR disulfide-linked dimer, labeled as 1 and 2 (Fig. 8A). We hypothesized that these two dimers harbor either one or two disulfide bonds between the opposing HypR subunits. The reversible oxidation to the intermolecular disulfide was also observed for the HypRC33A and HypRC142A mutant proteins under $\mathrm{NaOCl}$ and diamide stress, but not for the HypRC99A mutant (Fig. 8A, B, Supplementary Figs. S3 and S4). These results are in agreement with the in vitro DNA binding assays where the HypRC33A and HypRC142A mutants still responded to $\mathrm{NaOCl}$ and diamide stress leading to the relief of repression, while the HypRC99A mutant was impaired in DNA binding. However, the Cys33A mutant was less oxidized to intermolecular disulfides under diamide stress compared to $\mathrm{NaOCl}$, supporting that $\mathrm{Cys} 33$ is required for disulfide formation. In conclusion, our results show that HypR is oxidized to intermolecular disulfides under $\mathrm{NaOCl}$ and diamide stress, most likely involving Cys33 and Cys99 as the redox-sensing Cys residues in vitro.

We were interested to identify the Cys residues oxidized to the intermolecular disulfide in wild-type HypR under disulfide stress. The bands 1 and 2 of both oxidized HypR disulfides (Fig. 8A) were tryptic digested and subjected to matrixassisted laser desorption ionization-time of flight mass spectrometry (MALDI-TOF-MS) (Fig. 9). In the overview MS1 scan, a peptide was identified with the size of $3522.74 \mathrm{Da}$ corresponding to the size of the Cys33-SS-Cys99 intermolecular disulfide peptide (Fig. 9A). During fragmentation of this Cys33-SS-Cys99 peptide, the parent ion immediately disappeared and the disulfide bond fragmented into both single Cys33 and Cys99 peptides with the sizes of 1534.72 and 1992.90 Da (Fig. 9B). Moreover, fragment ions of both Cys peptides were observed without the sulfur and with the second sulfur atom that was engaged in the disulfide linkage in the parent ion. Thus, the fragmentation spectrum of this 3522.74 Da peptide is characteristic for the disulfide bond between the Cys33 and Cys99 peptides in oxidized wild-type HypR. Of note, the two oxidized HypR bands 1 and 2 in Figure 8A exhibited the same MS1 spectrum, supporting the idea of either one or two disulfide bonds between the HypR subunits (data not shown). 


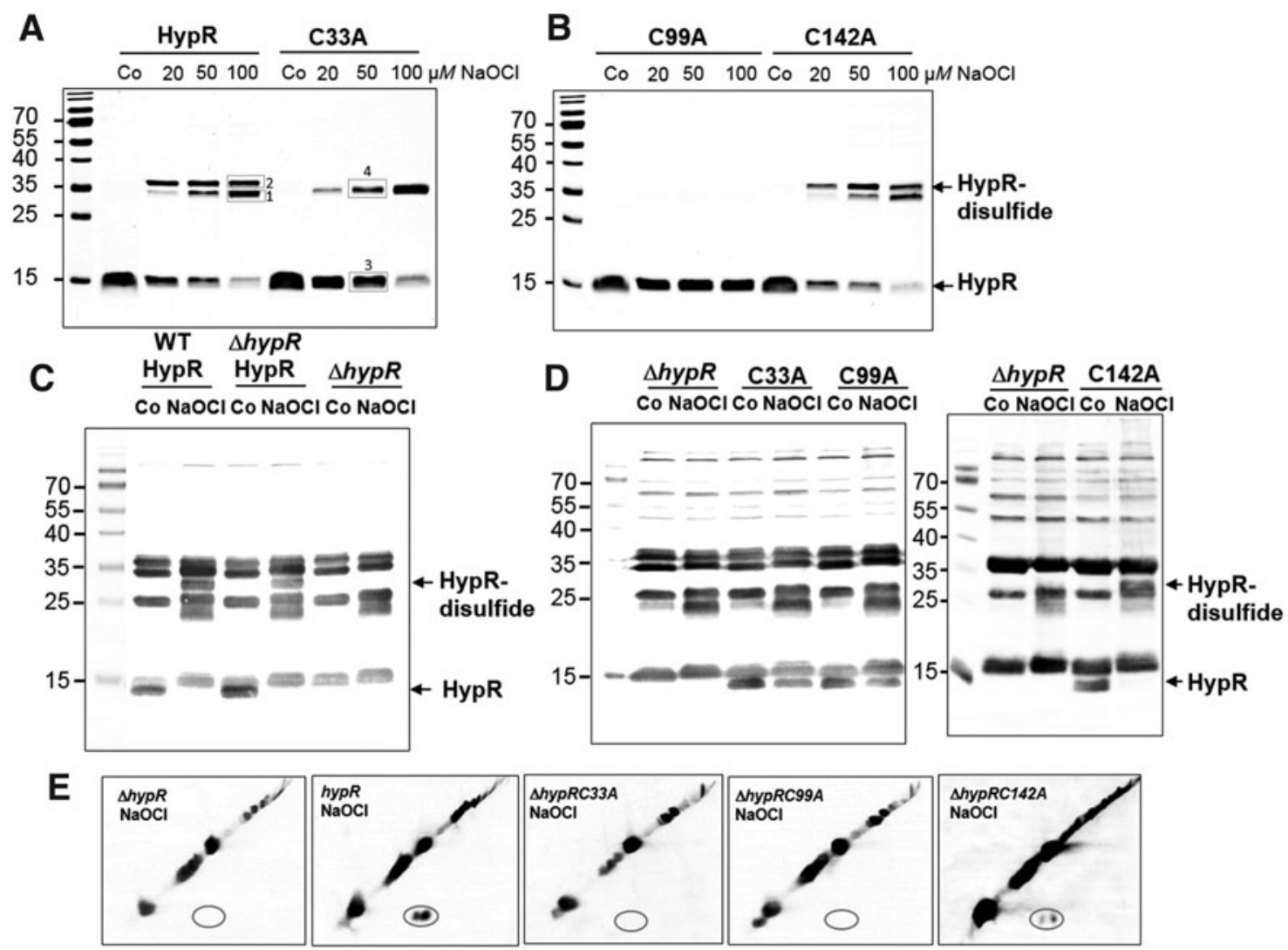

FIG. 8. HypR senses $\mathrm{NaOCl}$ stress by intermolecular disulfides, which requires Cys33 and Cys99 in vitro and in vivo. (A, B) The purified HypR wild-type and Cys mutant proteins were treated with increasing $\mathrm{NaOCl}$ concentrations in vitro and subjected to nonreducing SDS-PAGE analysis. The reduction of the HypR disulfides after DTT treatment is shown in the reducing SDS-PAGE analysis in Supplementary Figure S3. The HypR intermolecular disulfides (bands 1 and 2) were cut, tryptic digested, and subjected to MALDI-TOF MS/MS analysis as shown in Figure 9 to verify the Cys33Cys99 disulfide. The bands of the C33A mutant protein that were used for tryptic digestion and MS are boxed and labeled with 3 and 4. The MALDI-TOF results of the C33A mutant tryptic peptides are shown in Supplementary Figure S5B, C. (C, D) For the analysis of HypR disulfides in vivo, we used $S$. aureus COL with plasmid pRB473-hypR, the $\Delta h y p R$ deletion mutant and $\triangle$ hypR mutant strains complemented with hypR, hypRC33A, hypRC99A, and hypRC142A. S. aureus strains were exposed to $\mathrm{NaOCl}$ stress, alkylated with NEM, and protein extracts were subjected to nonreducing Western blot analysis using polyclonal rabbit anti-HypR antibodies. The reducing Western blot analysis of the HypR disulfides and loading controls is shown in Supplementary Figure S6. (E) Nonreducing/reducing diagonal SDS-PAGE and HypR-specific Western blot analysis of alkylated protein extracts were performed to verify the intersubunit disulfides for HypR and the HypR$\mathrm{C} 142 \mathrm{~A}$ mutant protein under $\mathrm{NaOCl}$ stress in vivo, but not in the HypRC33A and HypRC99A mutants. Additional diagonal assays using the HypR immunoprecipitates are shown in Supplementary Figure S7. MALDI-TOF-MS, matrix-assisted laser desorption ionization-time of flight mass spectrometry. NEM, N-ethylmaleimide.

In addition, we aimed to identify the disulfide crosslinked Cys residues in the Cys33A mutant that formed under $\mathrm{NaOCl}$ stress in vitro (Fig. 8A). MALDI-TOF-MS analysis revealed that Cys99 is fully reduced in the oxidized Cys33A mutant protein as shown by the iodoacetamide (IAM)-alkylated Cys99 peptide that was not detected in the oxidized HypR protein (Supplementary Fig. S5C, D). However, Cys142 could be not detected in the Cys33A mutant protein sample. These results suggest that HypR could be oxidized to the wrong Cys142-Cys142 disulfide that may crosslink two HypR dimers in vitro. The large peptide size of this putative Cys142Cys142 disulfide peptide excludes detection by MS.

Next, we used nonreducing Western blot analysis with polyclonal HypR antibodies to confirm the intermolecular disulfides between Cys33 and Cys99 in HypR under $\mathrm{NaOCl}$ stress in vivo. Protein extracts were prepared from the $S$. aureus COL wild type, the $\triangle h y p R$ mutant, and the hypR, hypRC33A, hypRC99A, and hypRC142A complemented strains under control and $\mathrm{NaOCl}$ stress, and the redox state of HypR was analyzed using nonreducing Western blots (Fig. 8C, D). Unfortunately, the HypR polyclonal antibodies showed cross-reactivity with other proteins of $S$. aureus which could be not be eliminated after preincubation of the antiserum with Western blots of the hypR mutant. However, the HypR specific bands could be clearly distinguished by using protein extracts from hypR mutant cells (Fig. 8C). The HypR protein was reduced in all controls of the hypR, hypRC33A, hypRC99A, and hypRC142A complemented strains (Fig. 8C, D). Under $\mathrm{NaOCl}$ stress, the oxidized HypR and HypRC142A mutant proteins migrated at the size of the intermolecular disulfide at $30 \mathrm{kDa}$, which was reversible with DTT as shown in the reducing SDS-PAGE (Supplementary 


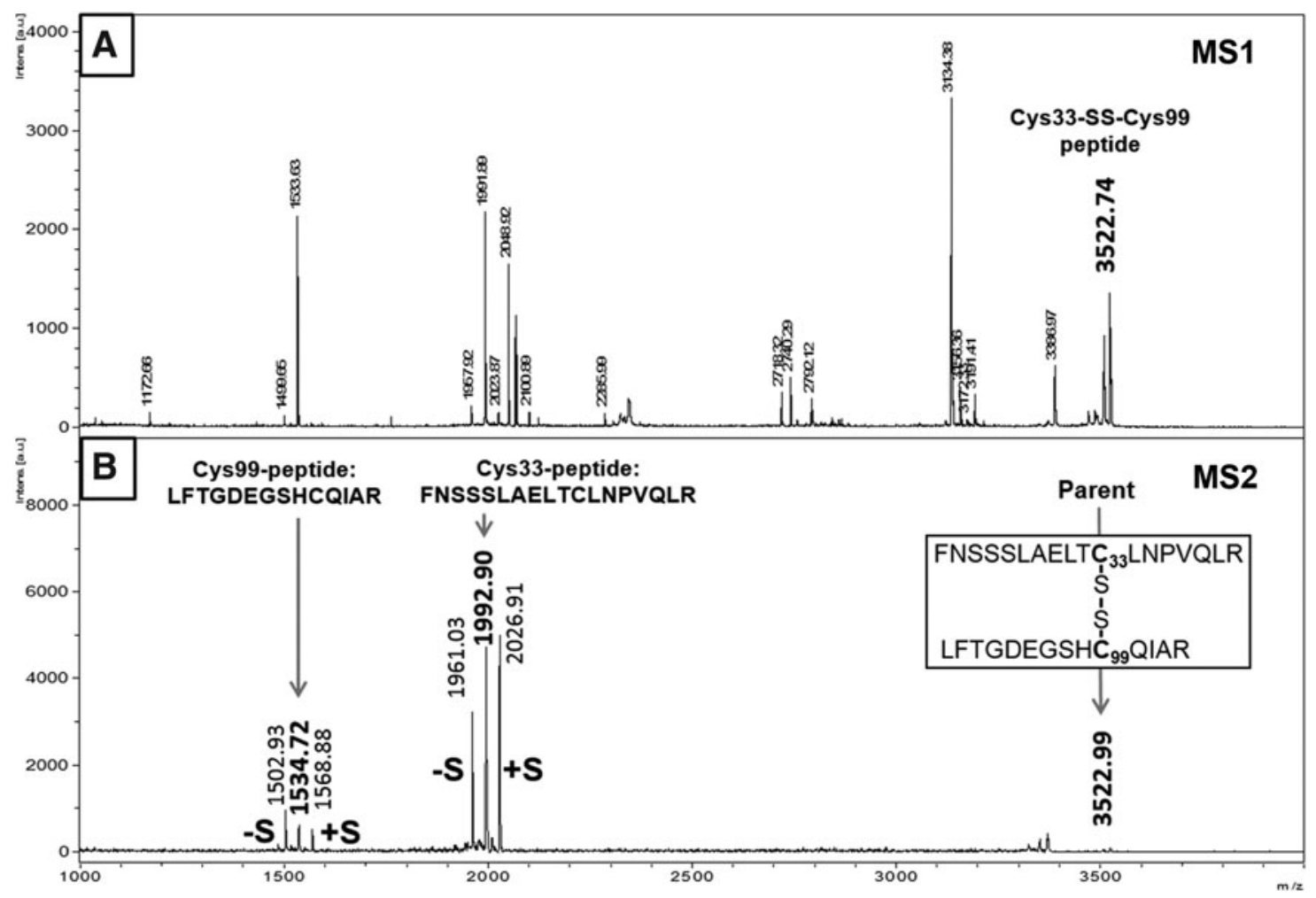

FIG. 9. HypR is oxidized to C33-C99' intermolecular disulfides in vitro as revealed by MALDI-TOF-TOF MS. The intermolecular disulfide band of the oxidized HypR wild-type protein of the SDS-PAGE in Figure 8A (band 1) was tryptic digested. The HypR peptides were measured by MALDI-TOF-TOF MS. (A) The upper panel indicates the MS1 overview scan of all peptides and (B) the lower panel shows the fragmentation of the C33-C99 disulfide peptide (3522.74 Da peak) into the Cys33 (1992.90 Da) and Cys99 peptides (1534.72 Da) in the MS2 scan. The parent ion of the disulfide peptide disappeared in the MS2 scan.

Fig. S6A, B). However, both Cys33A and Cys99A mutants did not form intermolecular disulfides under $\mathrm{NaOCl}$ exposure and failed to respond to $\mathrm{NaOCl}$ stress in vivo (Fig. 8D). These results are in agreement with the transcriptional data of hypRmerA expression and indicate that HypR responds to $\mathrm{NaOCl}$ stress by the formation of intersubunit disulfides between Cys33 and Cys99 in vivo and in vitro. To confirm the formation of the intermolecular disulfide in HypR and the involvement of Cys33 and Cys99 in the disulfide crosslink in vivo, diagonal nonreducing/reducing SDS-PAGE analyses were performed using the crude protein extracts and immunoprecipitated HypR. The diagonal assays clearly showed that HypR and the HypRC142A mutant protein migrate at the right side of the diagonal in $\mathrm{NaOCl}$-treated cells indicating intermolecular disulfide formation in vivo (Fig. 8E). In contrast, no HypR intermolecular disulfide was detected in the HypRC33A and HypRC99A mutant proteins in the diagonal assays (Fig. 8E and Supplementary Fig. S7). These data reveal that Cys33 and Cys99 are the redox-sensing Cys residues of HypR, required for disulfide bond formation both in vitro and in vivo.

\section{Analysis of the oligomerization states by size exclusion multiangle light scattering and circular dichroism spectroscopy of HypR and HypR Cys mutant proteins}

Cys99 is located at the end of the long $\alpha 6$ helix that forms the dimer interface in the HypR structure (Fig. 5B). The differences in the DNA-binding properties between HypR and the HypRC99A mutant protein raised the question whether the Cys99A mutation affects dimerization of HypR. Thus, we analyzed the oligomerization states of reduced HypR and HypR Cys-Ala mutant proteins using size exclusion chromatography/multiangle light scattering (SEC-MALS) (Supplementary Fig. S8). The results showed that all reduced proteins eluted mostly as dimers with a molecular weight of $\sim 32-34 \pm 2 \mathrm{kDa}$. Due to the redox sensitivity of Cys33 and Cys99, we observed higher molecular weight oligomeric species with molecular masses ranging from 67 to $78 \mathrm{kDa}$. The oligomer amounts differed between each Cys mutant (15-17\%) and might be related to disulfide crosslinks of two HypR dimers due to incomplete reduction of the proteins. Overall, the SEC-MALS results indicate that HypR and the three Cys-Ala mutant proteins are mostly present as dimers in solution.

Next, we used circular dichroism (CD) spectroscopy to compare the secondary structures between reduced HypR and the three Cys-Ala mutants to see whether the Cys99A mutation causes a conformational change. The far UV-CD spectra of HypR and the three Cys-Ala mutants revealed a significant $\alpha$-helical content in all proteins (Supplementary Fig. S9). The calculation of secondary structure elements using the program DichroWeb (http://dichroweb.cryst.bbk. ac.uk) $(86,94)$ showed that reduced HypR and all three CysAla mutants contain similar contents of $\alpha$-helices (34-37\%), $\beta$-sheets (11-13\%), and $\beta$-turns (18-19\%), confirming our 
structural model (Fig. 5B). Thus, under reduced conditions, the dimerization and secondary structure elements of HypR are retained in all three HypR Cys-Ala mutant proteins.

\section{HypR and MerA play important roles in the defense} of $\mathrm{S}$. aureus against $\mathrm{NaOCl}$ stress

Next, we analyzed the role of MerA in protection under $\mathrm{NaOCl}$ stress in $S$. aureus COL. MerA is annotated as nicotinamide adenine dinucleotide phosphate (NADPH)dependent FDR, which contains an active site Cys43 in a highly conserved $\mathrm{C}_{43} \mathrm{XXXXC}_{48}$ motif (Supplementary Fig. S10) (4). The active site Cys43 of MerA was also predicted in the THIOREDOXOME database (30). We hypothesized that MerA could be involved in reduction of cellular disulfides formed under oxidative stress and infection conditions. Thus, we analyzed the growth and survival of the $S$. aureus merA and hypR mutants and the complemented strains under $\mathrm{NaOCl}$ stress. The growth of the merA mutant was significantly impaired under sublethal $1.5 \mathrm{~m} M \mathrm{NaOCl}$ treatment in RPMI medium indicating that the merA mutant is more sensitive compared with the wild type (Fig. 10A). The NaOCl-sensitive growth defect of the merA mutant could be restored after complementation with merA expressed from plasmid pRB473 (Fig. 10B), but not when the active site Cys 43 of MerA was replaced by a serine. This indicates that MerA confers resistance to $\mathrm{NaOCl}$ and likely functions as defense mechanism under infection conditions in S. aureus. However, the hypR mutant was also slightly more sensitive to $1.75 \mathrm{mM} \mathrm{NaOCl}$ stress compared with the wild type suggesting that constitutive MerA expression does not confer a resistance phenotype (Fig. 10C, D).

We further studied the survival of the merA and hypR mutants and their complemented strains under $\mathrm{NaOCl}$ stress. Both merA and hypR mutants were impaired in their survival after exposure to lethal concentrations of $3.5 \mathrm{mM} \mathrm{NaOCl}$ (Fig. 11). The survival defect of the merA and hypR mutants could be restored back to wild-type level after complementation with merA and hypR, respectively. In agreement with the growth curves, Cys 43 of MerA was important for the function of MerA in the $\mathrm{NaOCl}$ stress defense in the survival assay. Moreover, the role of Cys33 for redox sensing of HypR was confirmed also in our survival phenotype assays.

\section{MerA and HypR are important for the survival of $\mathrm{S}$. aureus in macrophage infection assays}

Since the hypR-merA operon was most strongly induced in infection assays (91), we determined the survival of merA and hypR mutants inside murine macrophages in phagocytosis assays using the cell line J-774A.1. The macrophages were infected with $S$. aureus wild-type and mutant strains and the uptake of bacteria was stopped after $1 \mathrm{~h}$ with gentamycin to kill extracellular bacteria. The colony forming units (CFUs) of intracellular $S$. aureus were determined after 2,4 , and $24 \mathrm{~h}$ postinfection by plating serial dilutions of the host cell lysates.

After $4 \mathrm{~h}$ of infection, the CFUs of intracellular S. aureus cells were determined as approximately $10^{6}$ cells for the wild-
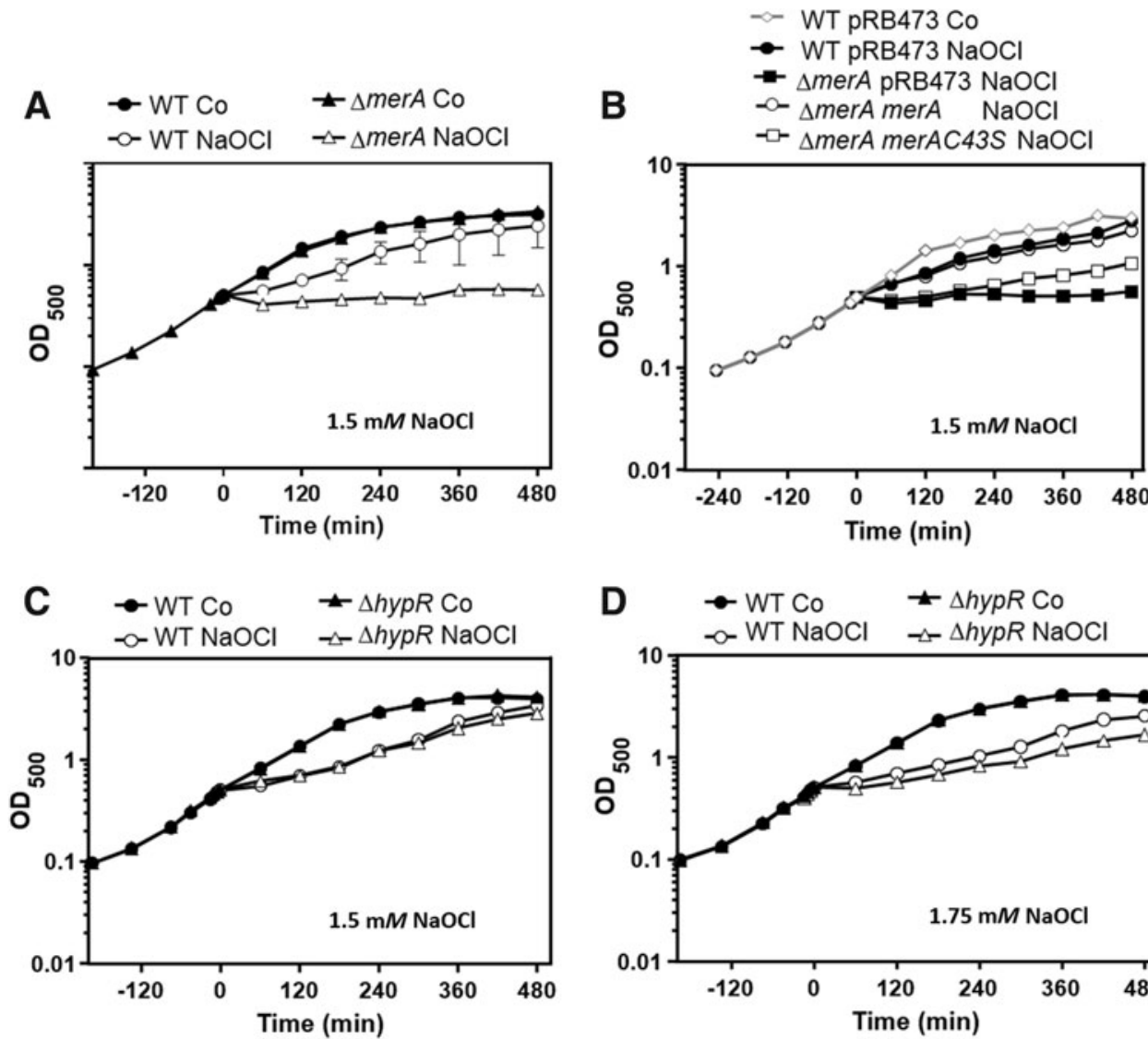

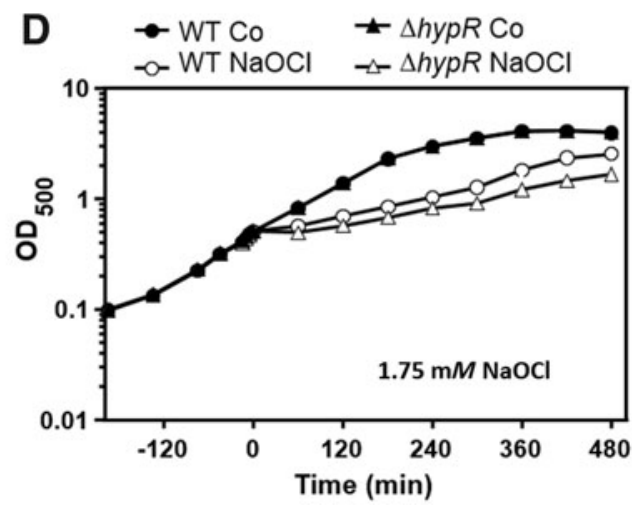

FIG. 10. The flavin disulfide reductase MerA is involved in the defense of $S$. aureus against hypochlorite stress. Growth phenotype analyses of the $S$. aureus wildtype (WT), the $\triangle m e r A$ mutant (A), the merA and merAC43S complemented $\triangle m e r A$ mutant strains (B) and the hypR mutant $(\mathbf{C}, \mathbf{D})$ before and after exposure to sublethal concentrations of 1.5 and $1.75 \mathrm{mM}$ $\mathrm{NaOCl}$ stress at an $\mathrm{OD}_{500}$ of 0.5. The NaOCl-sensitive growth phenotype of the merA mutant could be restored by complementation with plasmid-encoded merA and requires Cys43 in the MerAactive site. The results are from four biological replicate experiments. MerA, mercuric ion reductase; $\mathrm{OD}_{500}$, optical density at $500 \mathrm{~nm}$. 


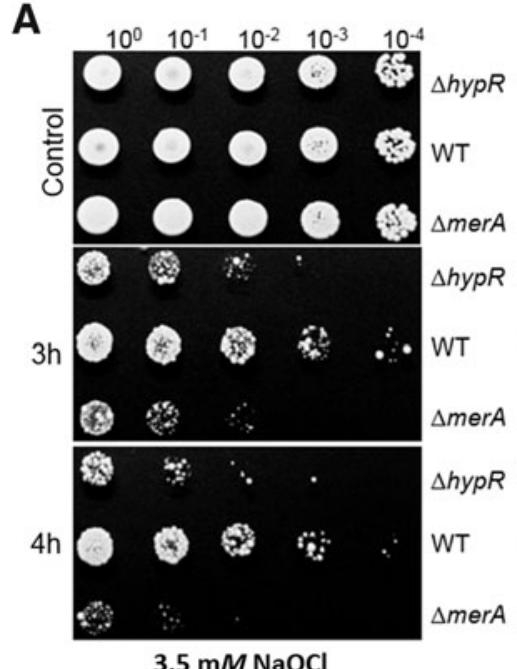

$3.5 \mathrm{mM} \mathrm{NaOCl}$

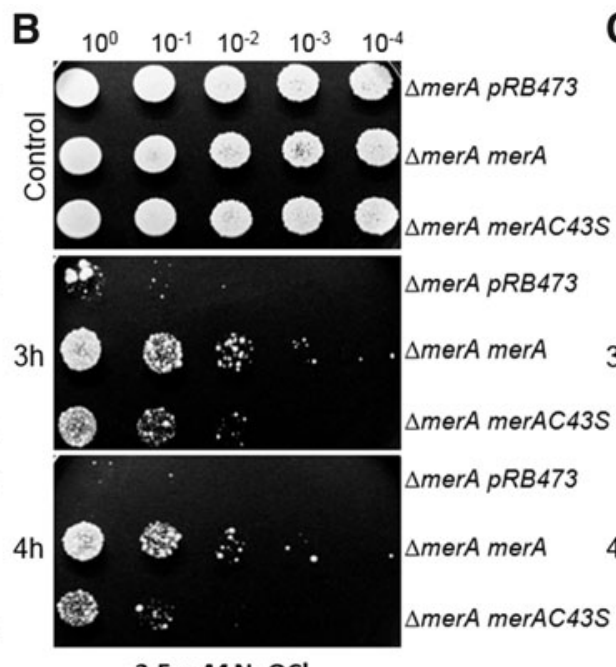

$3.5 \mathrm{~m} M \mathrm{NaOCl}$

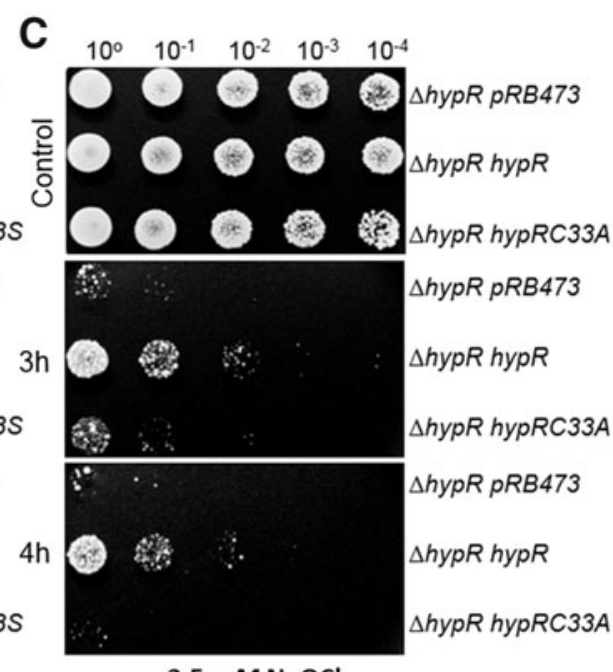

$3.5 \mathrm{mM} \mathrm{NaOCl}$

FIG. 11. MerA and HypR are both required for NaOCl stress survival in S. aureus. S. aureus COL wild-type (WT), $\triangle h y p R$ and $\triangle m e r A$ mutants (A), and their complemented strains and Cys mutants ( $\Delta$ merA merA, $\Delta$ merA merAC43S, $\Delta$ hypR hypR, ShypR hypRC33A) (B, C) were grown in RPMI until an $\mathrm{OD}_{500}$ of 0.5 and treated with $3.5 \mathrm{~m} M \mathrm{NaOCl}$. Survival assays were performed by spotting $10 \mu \mathrm{L}$ of serial dilutions after 3 and $4 \mathrm{~h}$ of $\mathrm{NaOCl}$ exposure onto LB agar plates. The active site Cys43 of MerA and the redox-sensing Cys33 of HypR are important for $\mathrm{NaOCl}$ stress survival. LB, Luria Bertani.

type, hypR, and merA mutants. However, $24 \mathrm{~h}$ after phagocytosis, the viable counts of intracellular $S$. aureus cells were decreased to $22.8 \%$ for the wild-type mutant and to $11.2 \%$ and $13.4 \%$ for the hypR and merA mutants, respectively (Fig. 12A). This indicates that merA and hypR mutants showed $40 \%$ and $50 \%$ decreased survival inside macrophages compared with the wild type after $24 \mathrm{~h}$ postinfection (Fig. 12C). This survival phenotype could be restored to $75-86 \%$ of wild-type level in both mutants after complementation with plasmid-encoded hypR and merA, respectively (Fig. 12B). These observations strongly indicate that MerA is required for the protection of $S$. aureus against the host innate immune defense under infection conditions. However, our results also show that constitutive expression of MerA in the hypR mutant does not increase intramacrophage survival. In conclusion, our in vitro growth and survival assays under $\mathrm{NaOCl}$ stress and the macrophage infection assays support that $\mathrm{MerA}$ is a major $\mathrm{NaOCl}$ defense mechanism enhancing survival of $S$. aureus in infection assays.

\section{Discussion}

During infections, $S$. aureus has to cope with the oxidative burst of activated macrophages and neutrophils, requiring a complex regulatory network of virulence and antibiotic regulators, for example, of the SarA/MarR family that senses ROS, RES, and RCS $(8,17,43)$. However, the redox-sensing mechanisms of $S$. aureus in response to strong bactericidal oxidants, such as hypochloric acid, are largely unknown. It is of utmost importance to understand the mechanisms of intracellular survival of $S$. aureus inside macrophages and neutrophils and the escape from destruction by oxidants to discover new drug targets to combat emerging drug-resistant S. aureus infections.

In this work, we have characterized the novel $\mathrm{NaOCl}-$ sensing transcription regulator HypR that belongs to the widely distributed Rrf2 family and controls the NADPH- dependent FDR MerA in S. aureus. The hypR-merA operon was previously highly induced during phagocytosis with neutrophils (91). In this work, we have used a global RNAseq analysis and identified the hypR-merA operon as most strongly upregulated under $\mathrm{NaOCl}$ stress. Detailed Northern blot analysis revealed that $\mathrm{NaOCl}$ and diamide stress specifically upregulates the hypR-merA operon, while sublethal concentrations of $\mathrm{H}_{2} \mathrm{O}_{2}$ and aldehydes do not lead to significant induction. Thus, HypR responds strongly to disulfide stress, but not to $\mathrm{H}_{2} \mathrm{O}_{2}$ and RES via intermolecular disulfide formation between Cys33 and Cys99. The sensitivity of $S$. aureus HypR to disulfide stress resembles the redoxsensing mechanisms of the MarR family regulators HypR and OhrR characterized in $B$. subtilis $(20,32,73)$ and of the TetR family regulator NemR from Escherichia coli $(35,36)$. These thiol-based regulators sense $\mathrm{HOCl}$, diamide, organic hydroperoxides, or electrophiles, such as N-ethylmaleimide (NEM) and methylglyoxal, and provide protection under $\mathrm{HOCl}$ stress. Since diamide is a very unspecific oxidant (49) that is not physiological relevant for $S$. aureus, we regard the Rrf2 regulator HypR as the most specific hypochlorite defense mechanism for $S$. aureus.

Some Rrf2 family transcriptional regulators contain FeS clusters, such as NsrR $(23,90,98)$ and $\operatorname{IcsR}(82,83)$, while others do not, such as CymR $(28,47,84)$ and the HypR homolog SaiR of $B$. anthracis (68). NsrR and IscR coordinate [4Fe-4S] clusters with three conserved Cys residues (Cys93, Cys99, and Cys106 in Sc-NsrR) present in the C-terminal domain (Fig. 5A, B) (90). However, only Cys99 in this Cterminal domain is conserved in HypR, while two other Cys residues are located in nonconserved positions at Cys 33 and Cys142 (Fig. 5A). Our results support a model that HypR senses and responds to $\mathrm{NaOCl}$ stress by intersubunit disulfide bond formation between Cys33 and Cys99 of the opposing subunits of the HypR dimer in vivo (Fig. 13). HypR oxidation leads to inactivation of its repressor function and upregulation of the FDR MerA. Thus, the S. aureus HypR repressor 

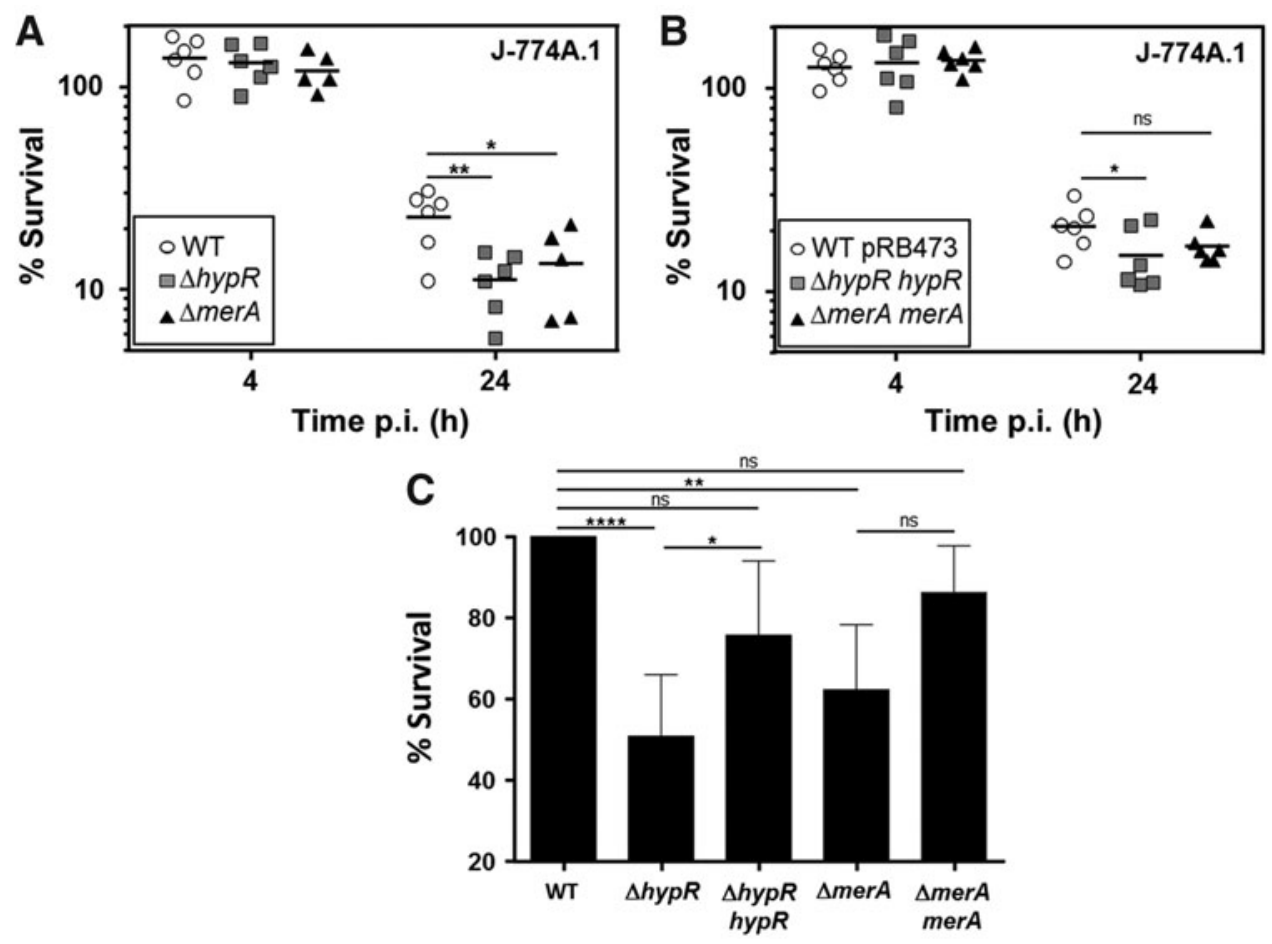

24h p.i.

FIG. 12. MerA and HypR are required for survival of $S$. aureus COL in murine macrophages. The survival of $S$. aureus strains was analyzed 2, 4, and $24 \mathrm{~h}$ postinfection (p.i.) of the murine macrophage cell line J-774A.1 and the CFUs were determined. $(\mathbf{A}, \mathbf{B})$ The percentages in survival of the $\Delta h y p R$ and $\Delta m e r A$ mutants and complemented strains were calculated in 5-6 biological replicate experiments and the survival at the 2-h time point was set to $100 \%$. (C) The average percentage in survival was calculated for each mutant and complemented strain in relation to the wild type (WT), which was set to $100 \%$. Results of 5-6 biological replicates are presented as scatter dots in (A, B) and mean values of percentage survival in comparison to the wild type (C). Error bars represent the SEM and the statistics was calculated using one-way ANOVA and Tukey's multiple comparisons post hoc test using the GraphPad Prism software. The $p$-values were deter-

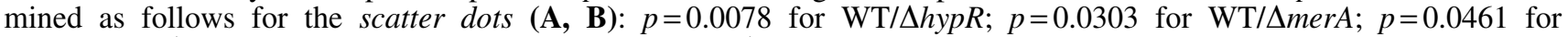

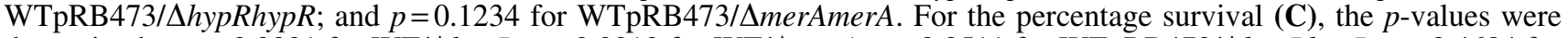

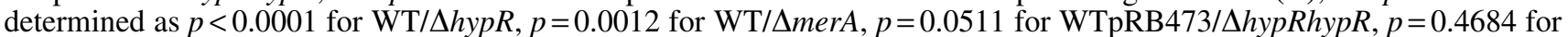

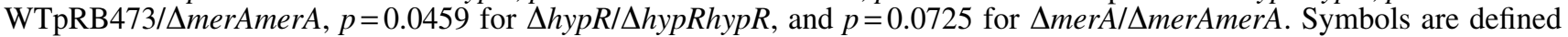
as follows: ${ }^{\mathrm{ns}} p>0.05 ; * p \leq 0.05 ; * * p \leq 0.01$; and $* * * * p \leq 0.0001$. CFU, colony-forming unit.

can be classified as typical two-Cys-type regulator since its mechanism of repressor inactivation resembles that of the two-Cys-type MarR/DUF24 family repressors HypR and YodB of $B$. subtilis that are redox sensors of $\mathrm{NaOCl}$, diamide, and quinones and inactivated via intersubunit disulfide formation $(18,73)$. The NemR repressor of $E$. coli was shown to sense $\mathrm{HOCl}$ and electrophiles, such as NEM and methylglyoxal, by intermolecular disulfide as well as sulfenamide formation $(34,36,50,72)$. Thiol oxidation of these repressors leads to conformational changes and dissociation of the repressors from their operator sites leading to derepression of their target genes.

The structural changes on oxidation have been first studied for the 2-Cys-type MarR/OhrR repressor of Xanthomonas campestris that senses organic hydroperoxides by Cys22-Cys127 intersubunit disulfide formation (51). OhrR oxidation breaks a conserved Cys22 hydrogen-bonding network and causes large structural rearrangements in the dimer interface and rigid body rotation of the DNA-binding domains causing dissociation from the DNA (51). Using mutational analysis, we showed that Cys33 and Cys99 of the
S. aureus HypR repressor are important for redox sensing of $\mathrm{NaOCl}$ and diamide stress in vivo, while Cys99 is required for repressor activity of HypR. Both Cys33 and Cys99 mutants did not form intermolecular disulfides in vivo as shown by nonreducing SDS-PAGE analysis. These results clearly support the two-Cys-type oxidation model of HypR for Cys33-Cys99 intermolecular disulfide bond formation. Thus, both Cys33 and Cys99 are required for redox sensing of $\mathrm{NaOCl}$ and are oxidized to the intersubunit disulfide in vitro and in vivo.

In comparison to other Rrf 2 transcription factors, HypR shows the highest sequence identity to SaiR of B. anthracis (20.4\%) and YwnA of B. subtilis (23.5\%) (Fig. 5A). Moreover, HypR and SaiR show common responses to disulfide stress (diamide, $\mathrm{NaOCl}$ ) and in infection assays inside macrophages in B. anthracis and $S$. aureus $(9,68)$. YwnA also responds to diamide stress (71) and probably controls the adjacent $y w n B$ gene encoding for an NADPH-binding reductase. SaiR of $B$. anthracis may play a related role like HypR of $S$. aureus in the protection against oxidative burst of activated macrophages and neutrophils under infection 


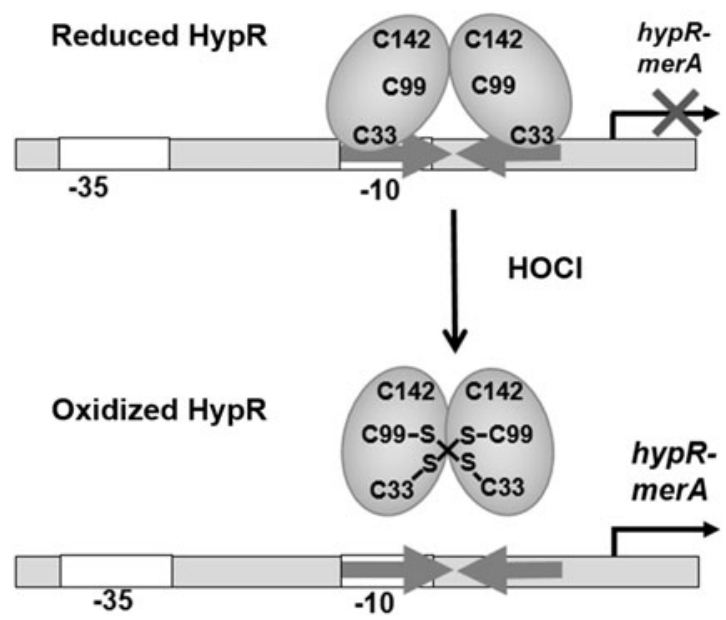

FIG. 13. Redox-sensing mechanism of HypR under hypochlorite stress during infection conditions in S. aureus. HypR controls the flavin disulfide reductase MerA, essential for growth and survival under hypochlorite stress and in macrophage infection assays in S. aureus. Cys33 and Cys99 of HypR are required for redox sensing in vivo. Under $\mathrm{NaOCl}$ stress, HypR is oxidized to Cys33-Cys99 intersubunit disulfides leading to derepression of hypR-merA transcription.

conditions. SaiR controls a five-gene operon that includes $s p x A 2$, saiR, and 3 unknown function genes (68). SpxA2 is a member of the ArsC (arsenate reductase) family that responds to $\mathrm{NaOCl}$ and diamide stress by thiol oxidation and interacts with the $\alpha$-C-terminal domain of the RNA polymerase to activate transcription of a large disulfide stress regulon $(69,70,99)$. SpxA2 together with its paralogue SpxA1 was shown to be required for diamide resistance and the saiR mutant conferred a peroxide-resistant phenotype (6, 68). Thus, SaiR of B. anthracis and HypR of $S$. aureus both confer protection under oxidative stress conditions in these pathogens via control of SpxA2 and MerA, respectively.

SaiR and HypR both share the conserved Cys96 and Cys99, respectively, which is required for repressor activity in vitro and in vivo as well as for redox sensing in vitro (68). To explain, why Cys 99 is required for DNA binding, the HypR structure was modeled based on the template of $B$. subtilis YwnA (1xd7) (Fig. 5A, B). The structural model suggests that Cys33 is located in the turn between the DNA recognition $\alpha 2$ and $\alpha 3$ helices, while the conserved Cys 99 is at the N-terminus of the long $\alpha 6$ helix that forms the highly hydrophobic dimer interface required for the elongated fold of Rrf2 family regulators (Fig. 5B). Recently, the structure of Streptomyces coelicolor NsrR was resolved with its [4Fe-4S] cluster in comparison to the three-Cys-Ala (3CA) mutant (90). Interestingly, the FeS cluster ligand Cys 99 is in close proximity to Gly37 of the $\alpha 3$ DNA recognition helix of the opposing subunit. In holo-NsrR, Gly37 forms a hydrogen bond with Asn97, which is interrupted in the 3CA mutant resulting in a shift of the $\alpha 3$ DNA binding helix (90). Gly37 of NsrR is the counterpart of Cys33 in HypR, suggesting that the Cys99A mutant also could break hydrogen bonds of Cys 33 leading to structural changes in the DNA-binding helices. This is supported by our mutational results since the C99A and Cys99S mutants are unable to bind DNA both in vitro and in vivo. The possible proximity of Cys33 and Cys99 in the structural model (based on the YwnA template) (Fig. 5B) further explains that both are involved in redox sensing of $\mathrm{NaOCl}$ and well positioned to form intersubunit disulfides as we confirmed by MS.

Our CD results further showed that HypR contains the predicted high $\alpha$-helical content. However, we could not show major structural changes in the secondary structure elements between HypR and the three Cys-Ala mutants. It will be interesting to further investigate the crystal structures of reduced and oxidized HypR proteins and the structural changes of the DNA-binding helices on oxidation.

Our gel shift assays and nonreducing SDS-PAGE and Western blot results further revealed that the Cys 33 mutant was impaired in redox sensing in vivo, but still responsive to $\mathrm{NaOCl}$ and diamide stress in vitro. However, compared to the wild type HypR protein, the Cys33A mutant showed a lower oxidation under diamide stress in the nonreducing SDS-PAGE in vitro. Since we could only detect reduced (alkylated) Cys99 and not the Cys 142 peptide in the mass spectrum of the intersubunit disulfide of the Cys33A mutant, the Cys33 mutant protein should be oxidized to the "wrong" "Cys142-Cys142" intersubunit disulfides, crosslinking two HypR dimers in vitro that also lead to HypR inactivation. However, this alternative oxidation was not observed in vivo, and thus, Cys33-Cys99 intersubunit disulfide formation is the physiologically relevant HOCl-sensing mechanism of HypR. Exposure of the purified Cys 33 mutant protein to low doses of $\mathrm{NaOCl}$ stress might have much stronger effects in vitro and this wrong disulfide might be not stabilized in vivo.

Another unanswered question is why merA and hypR mutants display both sensitivity to $\mathrm{NaOCl}$ stress and macrophage infections, significant phenotypes that could be restored by complementation with MerA and HypR. Our unpublished RNA-seq data of the wild-type and the hypR mutant identified only the hypR-merA operon as upregulated in the hypR mutant. Thus, the deficiency of MerA impaired the growth and survival under infections, and also its constitutive overproduction did not confer resistance. It might be possible that overproduction of MerA causes deficiency in the NADPH level, causing in turn a redox poise. The transcriptional results revealed a very low basal level of hypR-merA expression indicating that MerA expression is tightly regulated and its constitutive overexpression perhaps toxic for $S$. aureus explaining the $\mathrm{NaOCl}-$ sensitive phenotype of the hypR mutant.

However, our results clearly identify the NADPH-dependent disulfide reductase MerA as the major HOCl-defense mechanism of $S$. aureus. MerA also enhances the survival of S. aureus in J774A.1 murine macrophages during infection assays. The J774A.1 cell line was shown to produce NADPH oxidase and MPO during infections and on activation by drug treatment in vitro $(55,65)$. Increased ROS levels significantly contributed to the antimicrobial activity and killing of intracellular pathogens by the J774A.1 macrophage cell line (54). Thus, MerA could be involved in the defense of S. aureus against $\mathrm{ROS}$ and $\mathrm{HOCl}$ also during macrophage infections. Of note, there is a striking $49.2 \%$ sequence identity between MerA of $S$. aureus and the FDR RclA of E. coli that is controlled by the RCS-specific transcriptional activator RclR (75) (Supplementary Fig. S10). These conserved flavoenzymes may function as major defense mechanisms under hypochlorite stress in bacteria and are controlled by different redox regulators, the Rrf2 repressor HypR in S. aureus and the AraC activator RclR in E. coli (75). 
MerA is annotated as mercuric reductase, but it lacks the auxiliary C-terminal Cys residues that are important for $\mathrm{Hg}$ (II) reduction to elemental $\mathrm{Hg}^{0}$ in mercuric ion reductases $(4,63,64)$. Instead, MerA belongs to group-I FDR that uses a redox-active disulfide in the highly conserved $\mathrm{C}_{43} \mathrm{XXXXC}_{48}$ motif to transfer electrons from NADPH via an FAD cofactor to an unknown disulfide substrate (4). Our results have shown that the MerA-active site Cys43 is important for $\mathrm{NaOCl}$ survival since the merAC43S mutant was unable to complement the $\mathrm{NaOCl}$-sensitive phenotype of the merA mutant. The group-I FDR enzymes with known structures include glutathione disulfide reductase (Gor), thioredoxin reductase (TrxR), and mycothiol disulfide reductase (Mtr). We speculate that MerA could be involved in reduction of cellular LMW thiol/disulfides, such as oxidized bacillithiol disulfide (BSSB) or oxidized coenzymeA disulfide (CoAS2), that are increased under $\mathrm{NaOCl}$ stress. Our previous results showed an increased $\mathrm{BSH}$ redox potential using the genetically encoded Bacilliredoxin-redox-sensitive green fluorescent protein biosensor (58) and increased protein thiol oxidation under $\mathrm{NaOCl}$ stress in $S$. aureus indicating an increased BSSB level (46). Thus, it could be possible that MerA helps to reduce BSSB and CoAS2 to restore the redox balance. On the contrary, expression of MerA and HypR is coregulated, suggesting that both could constitute a redox couple and MerA could be involved in HypR reduction to regenerate the DNA-binding activity of HypR during recovery from $\mathrm{HOCl}$ stress. Our future studies are directed to identify the physiological disulfide substrate of MerA to understand the important role of this flavoenzyme in the defense against oxidative burst under macrophage and neutrophil infections.

\section{Experimental procedures}

Bacterial strains, growth, and survival assays. Bacterial strains, plasmids, and primers are listed in Supplementary Tables S3-S5. For cloning and genetic manipulation, E. coli was cultivated in Luria Bertani (LB) medium. For stress experiments, $S$. aureus USA300 and COL strains were cultivated in LB, RPMI, or BMM as described previously (58). Depending on the growth medium, different concentrations of $\mathrm{NaOCl}$ were applied due to the quenching effect of rich medium components, which affected the effective sublethal $\mathrm{NaOCl}$ doses. Thus, we applied sublethal $150 \mu M \mathrm{NaOCl}$ in BMM as described before (58) for RNA-seq analysis and $1-1.75 \mathrm{~m} M$ sublethal $\mathrm{NaOCl}$ in rich RPMI medium to analyze the expression and growth phenotype. For survival phenotype assays, $S$. aureus was grown in RPMI medium until an optical density at $500 \mathrm{~nm}\left(\mathrm{OD}_{500}\right)$ of 0.5 , exposed to a lethal dose of $3.5 \mathrm{mM} \mathrm{NaOCl}$ for $2-4 \mathrm{~h}$, and $10 \mu \mathrm{L}$ of serial dilutions were spotted onto LB agar plates for $24 \mathrm{~h}$. Complemented mutants that carried the pRB473 plasmid were grown in the presence of $1 \%$ xylose and $10 \mu \mathrm{g} / \mathrm{mL}$ chloramphenicol. Sodium hypochlorite, diamide, DTT, Tris(2-carboxyethyl)phosphine (TCEP), $\mathrm{H}_{2} \mathrm{O}_{2}$ (35\% w/v), formaldehyde, and methylglyoxal were purchased from Sigma Aldrich.

RNA isolation, library preparation, and next-generation cDNA sequencing. S. aureus USA300 was cultivated in BMM and treated with $150 \mu M \mathrm{NaOCl}$ stress as described previously (58). S. aureus cells were harvested before (as untreated control) and $30 \mathrm{~min}$ after exposure to $150 \mu \mathrm{M} \mathrm{NaOCl}$ and disrupted in $3 \mathrm{~m} M$ ethylenediaminetetraacetic acid (EDTA)/ $200 \mathrm{~m} M \mathrm{NaCl}$ lysis buffer with a Precellys 24 ribolyzer. RNA isolation was performed using the acid phenol extraction protocol as described (19). The RNA quality was checked by Trinean Xpose (Gentbrugge, Belgium) and the Agilent RNA Nano 6000 kit using an Agilent 2100 Bioanalyzer (Agilent Technologies, Böblingen, Germany). Ribo-Zero rRNA Removal Kit (Bacteria) from Illumina (San Diego, CA) was used to remove the rRNA. TruSeq Stranded mRNA Library Prep Kit from Illumina was applied to prepare the cDNA libraries. The cDNAs were sequenced paired end on an Illumina HiSeq 1500 and MiSeq system (San Diego, CA) using 50 and $70 \mathrm{bp}$ read length. The transcriptome sequencing raw data files are available in the ArrayExpress database under accession number: E-MTAB-5666.

Bioinformatics data analysis, read mapping, data visualization, and analysis of differential gene expression. Trimmed reads (26 nt) were mapped to the S. aureus USA300_TCH1516 genome sequence (accession number CP000730) (41) using SARUMAN (11), allowing one error per read. The forward and reverse reads, if both present and with a maximum distance of $1 \mathrm{~kb}$, were combined to one read containing the reference sequence as insert. Paired mappings with a distance $>1 \mathrm{~kb}$ were discarded, and paired reads with either only the forward or the reverse read mapping were retained as single mapping reads. For the visualization and counting of short read alignments, ReadXplorer v2.2 (42) was used.

Differential gene expression analysis, including normalization, was performed using Bioconductor package DESeq2 (59) included in the ReadXplorer v2.2 software (42). The signal intensity value (a-value) was calculated by $\log 2$ mean of normalized read counts and the signal intensity ratio (m-value) by $\log 2$ fold change. The evaluation of the differential RNA-seq data was performed using an adjusted $\mathrm{p}$-value cutoff of $p \leq 0.05$ and a signal intensity ratio (m-value) cutoff of $\geq 1.98$ or $\leq-1.98$. The latter was determined by applying a significance level of 5\% to the experiment with the assumption that the majority of genes are not differentially transcribed. Thus, $95 \%$ of all $\mathrm{m}$-values should fall in this range. Therefore, the standard deviation (STDEV) for all $\mathrm{m}$-values was calculated and the cutoff was set to $\mathrm{m}=1.96 *$ STDEV. Genes with an $\mathrm{m}$-value outside this range and $p \leq 0.05$ were considered as differentially transcribed.

Construction of Voronoi treemaps. The software Paver (DECODON GmbH, Greifswald, Germany) was used to generate the regulon treemap showing $\log 2$ fold changes (m-values) of selective genes sorted into operons and regulons that are upregulated under $\mathrm{NaOCl}$ stress compared to the untreated control. The treemap construction is based on a model of competing particle swarms for the layout optimization as described (62). The hierarchical structure of the treemap is defined by the regulons (on the first level-bold white labels), genes, and operons (second level-small contrast optimized labels). Cell sizes are defined as absolute $\log 2$ fold changes of expression levels after $\mathrm{NaOCl}$ stress divided by the untreated control. Colors are defined by a symmetric divergent color gradient (negative valuesblue and positive values orange - red) of $\mathrm{NaOCl}$ versus control $\log 2$ fold ratios. 
Cloning, expression, and purification of His-tagged HypR and HypR Cys mutant proteins in E. coli. The hypR gene (SACOL0641) was amplified from chromosomal DNA of S. aureus COL by PCR using primers 0641-pET-for-NheI and 0641-pET-rev-BamHI (Supplementary Table S5), digested with NheI and BamHI and inserted into plasmid pET11b (Novagen) that was digested using the same restriction enzymes to generate plasmid pET11b-hypR.

For construction of HypR Cys-to-Ala and Cys-to-Ser variants, the Cys residues of HypR were replaced by alanine or serine, respectively, using PCR mutagenesis. For the hypRC33A mutant, two first-round PCRs were performed using primers 0641-pET-for-NheI and 0641-pET-C33A-Rev and primers 0641-pET-C33A-for and 0641-pET-rev-BamHI (Supplementary Table S5). For the hypRC99A mutant, two first-round PCRs were performed using primers 0641-pETfor-NheI and 0641-pET-C99A-rev and primers 0641-pETC99A-for and 0641-pET-rev-BamHI. For the hypRC99S mutant, two first-round PCRs were performed using primers 0641-pET-for-NheI and 0641-pET-C99S-rev and primers 0641-pET-C99S-for and 0641-pET-rev-BamHI. The two PCR products of each first-round PCR were hybridized and subsequently amplified by a second round of PCRs using primers 0641-pET-for-NheI and 0641-pET-rev-BamHI. The PCR products from the second-round PCRs were digested with NheI and BamHI and inserted into plasmid pET11b digested with the same enzymes to generate plasmids pET11b-hypRC33A, pET11b-hypRC99A, and pET11b-hypRC99S. The hypRC142A gene was amplified from chromosomal DNA of S. aureus COL by PCR using primers 0641-pET-for-NheI and 0641-pET-C142A-rev-BamHI, digested with NheI and BamHI and inserted into plasmid pET11b that was digested using the same restriction enzymes to generate plasmid pET11b-hypR$C 142 A$. The correct sequences of all inserts were confirmed by PCR and DNA sequencing.

For expression and purification of $\mathrm{His}_{6}$-tagged HypR wildtype and Cys mutant proteins, E. coli BL21(DE3) plysS was used that contains the plasmids pET11b-hypR, pET11bhypRC33A, pET11b-hypRC99A, pET11b-hypRC99S, and pET11b-hypRC142A. These E. coli expression strains were cultivated in $11 \mathrm{LB}$ medium until the exponential growth phase at $\mathrm{OD}_{600}$ of 0.8 followed by addition of $1 \mathrm{mM}$ isopropyl$\beta$-D-thiogalactopyranoside for $3.5 \mathrm{~h}$ at $37^{\circ} \mathrm{C}$. E. coli strains expressing recombinant $\mathrm{His}_{6}$-tagged HypR and the HypR Cys mutant proteins were disrupted by sonication in binding buffer (20 mM NaH${ }_{2} \mathrm{PO}_{4}, 500 \mathrm{mM} \mathrm{NaCl}, 20 \mathrm{~m} M$ imidazole, $\mathrm{pH}$ 7.4). Lysates were cleared from cell debris by repeated centrifugation, and purification of the His-tagged proteins was performed using His Trap ${ }^{\mathrm{TM}} \mathrm{HP}$ Ni-NTA columns $(5 \mathrm{~mL}$; GE Healthcare, Chalfont St Giles, United Kingdom) and the ÄKTA purifier liquid chromatography system (GE Healthcare) according to the instructions of the manufacturer. Supplementary Figure S11 shows the HypR purification profile in the Äkta chromatography. A gradient from 0 to $500 \mathrm{mM}$ imidazole was applied in elution buffer $\left(20 \mathrm{mM} \mathrm{NaH} \mathrm{NaO}_{4}\right.$, $500 \mathrm{~m} M \mathrm{NaCl}, 500 \mathrm{~m} M$ imidazole, $\mathrm{pH}$ 7.4) and HypR eluted at $\sim 240 \mathrm{~m} M$ imidazole. The purity of eluted and pooled fractions was analyzed by reducing SDS-PAGE (Supplementary Fig. S11, inset). The purified proteins were extensively dialyzed against $10 \mathrm{mM}$ Tris- $\mathrm{HCl}(\mathrm{pH} 8.0), 100 \mathrm{mM} \mathrm{NaCl}$, and $30 \%$ glycerol and stored at $-80^{\circ} \mathrm{C}$. The HypR protein concentrations were determined by UV absorbance using a
Thermo Nanodrop 2000c spectrophotometer (Thermo Scientific, Germany). The final HypR protein concentration after dialysis was $2.8 \mathrm{mg} / \mathrm{mL}(158.7 \mu M)$ with a threefold enrichment after dialysis. Before further usage, HypR proteins were freshly reduced with $10 \mathrm{~m} M$ DTT or $10 \mathrm{mM}$ TCEP or as otherwise stated in the methods and figure legends.

Construction of $S$. aureus COL hypR and merA deletion mutants and complemented Cys-Ala mutant strains. $S$. aureus COL mutants with clean deletions of hypR (SACOL0641) and merA (SACOL0640) were constructed by allelic replacement via the temperature-sensitive shuttle vector pMAD as described previously (5). Briefly, for construction of the plasmids, pMAD- $\Delta$ hypR and pMAD- $\Delta m e r A$, the flanking gene regions, including $500 \mathrm{bp}$ upstream and downstream of hypR and merA of S. aureus COL, were amplified using the primers SACOL0641-pMAD and SACOL0640-pMAD (Supplementary Table S5). The upand downstream flanking regions of hypR and merA were each fused by overlap extension PCR and ligated into the $B g l I I$ and SalI sites of plasmid pMAD. The pMAD constructs were electroporated into the restriction-negative and methylation-positive intermediate $S$. aureus RN4220 strain and further transferred to $S$. aureus $\mathrm{COL}$ by phage transduction using phage $80(81)$. Transductants were streaked out on LB agar with $10 \mu \mathrm{g} / \mathrm{mL}$ erythromycin and $40 \mu \mathrm{g} / \mathrm{mL}$ 5-bromo-4-chloro-3-indolyl- $\beta$-D-galactopyranoside (X-gal) at $30^{\circ} \mathrm{C}$. Blue transductants with pMAD integrations were used for plasmid excision by a heat shock and screened for erythromycin-sensitive white colonies on X-gal plates as described (66). The clean deletions of internal gene regions of hypR or merA were confirmed by PCR and sequencing.

The complemented hypR and merA deletion mutants and Cys mutant strains were constructed using the pRB473-XylR plasmid as described previously (58). Briefly, hypR, hypRC33A, hypRC99A, hypRC142A, merA, and merAC43S sequences were amplified from the $E$. coli expression plasmids pET11b-hypR, pET11b-hypRC33A, pET11b-hypRC99A, pET11b-hypRC142A, pET11b-merA, and pET11b-mer$A C 43 S$ using the following primers. Primers 0641-pRB-forBamHI and 0641-pRB-REV-KpnI were used to construct plasmids pRB473-XylR-hypR, pRB473-XylR-hypRC33A, and pRB473-XylR-hypRC99A. Primers 0641-pRB-forBamHI and 0641-pRB-REV-KpnI-C2-3A were used to construct pRB473-XylR-hypRC142A. Primers 0640-pRBfor-BamHI and 0640-pRB-rev-KpnI were used to construct pRB473-XylR-merA and pRB473-XylR-merAC43S. Each forward primer also includes the shine-dalgarno sequence of hypR or merA, respectively. The PCR product was digested with BamHI and $K p n I$ and inserted into the pRB473-XylR shuttle vector that was digested using the same enzymes. The recombinant plasmids were introduced into the $\triangle h y p R$ or $\Delta$ merA deletion mutants via phage transduction as described previously (58).

Northern blot experiments. Northern blot analyses were performed as described previously (93) using RNA isolated from the $S$. aureus COL strains that were exposed to $1 \mathrm{mM}$

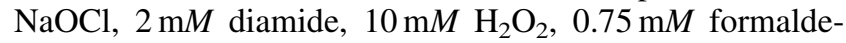
hyde, and $0.5 \mathrm{~m} M$ methylglyoxal (MG) for 15 and $30 \mathrm{~min}$ as indicated. Hybridizations were performed with the digoxigenin-labeled hypR-and merA-specific antisense RNA 
probes synthesized in vitro using T7 RNA polymerase as described previously (89). The primer pairs SACOL0641-for and SACOL0641-rev or SACOL0640-for and SACOL0640rev were each used for generation of the digoxygenin-labeled hypR and merA antisense RNA probes, respectively.

EMSAs of HypR. For EMSAs, we amplified a DNA fragment containing the hypR-merA upstream region that covered the region from -128 to +70 relative to the transcriptional start site using PCR with the primer set emsa0641-for and emsa0641-rev (Supplementary Table S5). Approximately $0.75 \mathrm{ng}$ of purified PCR products was incubated with different amounts of purified His-HypR and His-HypR Cys mutant proteins for $45 \mathrm{~min}$ at room temperature in EMSA binding buffer (10 m $M$ Tris-HCl [pH 7.5], $100 \mathrm{mM} \mathrm{KCl,} \mathrm{5 \%} \mathrm{glycerol,}$ $50 \mu \mathrm{g} / \mathrm{mL}$ bovine serum albumin (BSA), and $5 \mu \mathrm{g} / \mathrm{mL}$ salmon sperm DNA) in the presence of $10 \mathrm{~m} M$ DTT. To study the effect of $\mathrm{NaOCl}$ and diamide on the DNA-binding activity in vitro, the purified proteins were reduced with $10 \mathrm{~m} M$ DTT for $20 \mathrm{~min}$, desalted with Micro-Bio spin columns (Biorad), and diluted to $0.6 \mu M$ in $10 \mathrm{~m} M$ Tris- $\mathrm{HCl}$, pH 7.5. Reduced HypR and Cys mutant proteins were oxidized with 1.25-20 $\mu M$ $\mathrm{NaOCl}$ or 2.5-20 $\mu M$ diamide for $10 \mathrm{~min}$ at room temperature and incubated with the DNA fragment $(0.75 \mathrm{ng})$ containing the hypR-merA promoter. DNA-binding reactions were separated by $4 \%$ native polyacrylamide gel electrophoresis in $10 \mathrm{mM}$ Tris, $1 \mathrm{~m} M$ EDTA buffer, $\mathrm{pH} 8$, containing $2.5 \%$ glycerol at room temperature and constant voltage $(180 \mathrm{~V})$ for $30 \mathrm{~min}$. Gels were stained with SYBR green (Thermo Fisher Scientific) for $30 \mathrm{~min}$ in the dark and fluorescence was visualized using a Typhoon scanner (Typhoon FLA 9500; GE Healthcare Life Sciences).

CD spectroscopy. CD spectra of DTT-reduced HypR and HypR Cys mutant proteins were obtained using a Jasco J-810 spectropolarimeter with a HAAKE WKL recirculating chiller (D-76227, Karlsruhe). The DTT-reduced proteins were measured at $10 \mu M$ in $20 \mathrm{~m} M$ potassium phosphate buffer, $\mathrm{pH} 7.5$, with $1 \mathrm{~m} M$ DTT. The quartz cuvettes $(2 \mathrm{~mm}$ path length, Suprasil Hellma) were set at a constant temperature of $25^{\circ} \mathrm{C}$ with a Jasco PTC-423S Peltier-type thermocouple. Secondary structure elements were calculated using the program DichroWeb (http://dichroweb.cryst.bbk.ac.uk) $(86,94)$.

SEC-MALS analysis. HypR proteins were diluted in SEC buffer (10 m $M$ HEPES, $500 \mathrm{~m} M \mathrm{NaCl}$, pH 7.4, $1 \mathrm{~m} M$ TCEP) and reduced with $10 \mathrm{~m} M$ TCEP at $25^{\circ} \mathrm{C}$ for $1 \mathrm{~h}$. The molecular weights and oligomerization states of HypR and the HypR Cys mutants were determined using SEC-MALS analysis at $20^{\circ} \mathrm{C}$ running in SEC buffer. The reduced HypR protein samples (170 $\mu M$ each) were passed over a Superdex 75 10/300 size-exclusion column (GE Healthcare) coupled to a miniDAWN TREOS three-angle light scattering detector (Wyatt Technology) and a RefractoMax520 refractive index detector (ERC). Detectors were aligned, corrected for band broadening, and the photodiodes of the miniDAWN TREOS were normalized with BSA as a reference. Data were analyzed with ASTRA 6.1.4.25 (Wyatt Technology). For calculation of the molecular masses, protein concentrations were determined from the differential refractive index with a specific refractive index increment $(\mathrm{dn} / \mathrm{dc})$ of $0.185 \mathrm{~mL} / \mathrm{g}$.
Nonreducing/reducing diagonal SDS-PAGE analysis. $S$. aureus COL strains were cultivated in BMM, treated with $150 \mu M \mathrm{NaOCl}$ for $30 \mathrm{~min}$ as described (58), harvested, and washed in TE-buffer ( $10 \mathrm{~m} M$ Tris- $\mathrm{HCl}, \mathrm{pH} 8 ; 1 \mathrm{~m} M$ EDTA) with $50 \mathrm{~m} M$ NEM to alkylate reduced thiols. Cells were disrupted using the ribolyzer, and the protein extracts were obtained after repeated centrifugation. Immunoprecipitation of HypR proteins was performed using HypR-polyclonal antibodies and $S$. aureus protein extracts with Dynabeads protein A (Invitrogen) according to the instructions of the manufacturer. The precipitated HypR proteins were eluted in nonreducing SDS sample buffer (2\% SDS, $62.5 \mathrm{mM}$ Tris- $\mathrm{HCl}$ [pH 8.0]). The protein extracts and immunoprecipitated HypR proteins were separated by $15 \%$ nonreducing/reducing SDS-PAGE as described previously (53). In the first SDSPAGE, proteins were separated in nonreducing SDS-PAGE sample buffer. The lanes were cut and incubated in reducing SDS sample buffer (with $50 \mathrm{~m} M$ DTT) followed by thiol alkylation with $50 \mathrm{~m} M$ iodoacetamide (IAM) for each $15 \mathrm{~min}$. The bands were positioned horizontally on an SDS-PAGE gel, separated using reducing SDS-PAGE, and subjected to HypR-specific Western blot analysis as described previously (22). HypR forms intermolecular disulfides between two subunits that run at the right side of the diagonal.

Western blot analysis. S. aureus COL cells were harvested, washed in TE buffer ( $\mathrm{pH}$ 8.0) with $50 \mathrm{~m} M$ NEM, disrupted using the ribolyzer, and the protein extract was cleared from cell debris by repeated centrifugation as described above. Protein amounts of $25 \mu \mathrm{g}$ were diluted in nonreducing or reducing SDS sample buffer (as above), separated using 15\% SDS-PAGE, and the Western blot analysis was performed as described previously (22). Anti-HypR polyclonal rabbit antiserum was generated using purified His-HypR protein and used at a dilution of 1:500 for Western blot analyses.

MALDI-TOF-MS of in vitro oxidized HypR protein. The purified HypR and HypRC33A proteins were oxidized with $\mathrm{NaOCl}$ and all reduced thiols were blocked with $50 \mathrm{~m} M$ IAM resulting in the mass increase of $57 \mathrm{Da}$ at Cys residues. The oxidized HypR and HypRC33A intermolecular disulfides were separated by nonreducing SDS-PAGE, followed by tryptic digestion of the HypR and HypRC33A disulfide bands as described (21). The peptides were measured using a MALDITOF-MS using an Ultraflex-II TOF/TOF instrument (Bruker Daltonics, Bremen, Germany) equipped with a $200 \mathrm{~Hz}$ solidstate Smart beam ${ }^{\mathrm{TM}}$ laser. The mass spectrometer was operated in the positive reflector mode. Mass spectra were acquired over an $\mathrm{m} / \mathrm{z}$ range of 600-4000. MS/MS spectra of selected peptides were acquired in the LIFT mode as described previously (87).

Infection assays with murine macrophage cell line $\mathrm{J}$ 774A.1. Murine macrophage cell line J-774A.1 (80) was cultivated in Iscove's modified Dulbecco's medium (Biochrom) with $10 \%$ heat-inactivated fetal bovine serum (FBS) and seeded in cell culture dishes for 2 days under $5 \% \mathrm{CO}_{2}$ at $37^{\circ} \mathrm{C}$ as described (79). Before the infection assay, S. aureus COL strains were plated overnight on LB agar and resuspended in eukaryotic growth medium. Macrophages were infected with $S$. aureus cells at a multiplicity of infection (MOI) of 1:25. One hour after infection, the cell culture medium was replaced and $150 \mu \mathrm{g} / \mathrm{mL}$ gentamycin was added 
for $1 \mathrm{~h}$ to kill extracellular bacteria and to stop the uptake of $S$. aureus. The intracellular survival was determined at 2,4 , and $24 \mathrm{~h}$ after phagocytosis. Infected macrophages were lysed with $0.1 \%$ Triton $\mathrm{X}-100$ and the supernatant with internalized intracellular bacteria was plated on agar plates for CFUs.

\section{Acknowledgments}

We are grateful to Dorian Jamal Mikolajczak and Prof. Beate Koksch (Organic chemistry, FU Berlin) for support with CD spectrometry. This work was supported by an ERC Consolidator grant (GA 615585) MYCOTHIOLOME and grants from the Deutsche Forschungsgemeinschaft (AN746/41 and AN746/4-2) within the SPP1710 on "Thiol-based Redox switches" as well as by the SFB973 project C08 to H.A.

\section{Author Disclosure Statement}

No competing financial interests exist.

\section{References}

1. Abomoelak B, Hoye EA, Chi J, Marcus SA, Laval F, Bannantine JP, Ward SK, Daffe M, Liu HD, and Talaat AM. mosR, a novel transcriptional regulator of hypoxia and virulence in Mycobacterium tuberculosis. J Bacteriol 191: 5941-5952, 2009.

2. Antelmann H and Helmann JD. Thiol-based redox switches and gene regulation. Antioxid Redox Signal 14: 1049-1063, 2011.

3. Archer GL. Staphylococcus aureus: a well-armed pathogen. Clin Infect Dis 26: 1179-1181, 1998.

4. Argyrou A and Blanchard JS. Flavoprotein disulfide reductases: advances in chemistry and function. Prog Nucleic Acid Res Mol Biol 78: 89-142, 2004.

5. Arnaud M, Chastanet A, and Debarbouille M. New vector for efficient allelic replacement in naturally nontransformable, low-GC-content, gram-positive bacteria. Appl Environ Microbiol 70: 6887-6891, 2004.

6. Barendt S, Lee H, Birch C, Nakano MM, Jones M, and Zuber P. Transcriptomic and phenotypic analysis of paralogous spx gene function in Bacillus anthracis sterne. Microbiologyopen 2: 695-714, 2013.

7. Baroja ML, Herfst CA, Kasper KJ, Xu SX, Gillett DA, Li J, Reid G, and McCormick JK. The SaeRS two-component system is a direct and dominant transcriptional activator of toxic shock syndrome toxin 1 in Staphylococcus aureus. J Bacteriol 198: 2732-2742, 2016.

8. Beavers WN and Skaar EP. Neutrophil-generated oxidative stress and protein damage in Staphylococcus aureus. Pathog Dis 74: pii: ftw060, 2016.

9. Bergman NH, Anderson EC, Swenson EE, Janes BK, Fisher N, Niemeyer MM, Miyoshi AD, and Hanna PC. Transcriptional profiling of Bacillus anthracis during infection of host macrophages. Infect Immun 75: 3434-3444, 2007.

10. Biasini M, Bienert S, Waterhouse A, Arnold K, Studer G, Schmidt T, Kiefer F, Gallo Cassarino T, Bertoni M, Bordoli L, and Schwede T. SWISS-MODEL: modelling protein tertiary and quaternary structure using evolutionary information. Nucleic Acids Res 42: W252-W258, 2014.

11. Blom J, Jakobi T, Doppmeier D, Jaenicke S, Kalinowski J, Stoye J, and Goesmann A. Exact and complete short-read alignment to microbial genomes using graphics processing unit programming. Bioinformatics 27: 1351-1358, 2011.
12. Boucher HW and Corey GR. Epidemiology of methicillinresistant Staphylococcus aureus. Clin Infect Dis 46 Suppl 5: S344-S349, 2008.

13. Chandrangsu P, Loi VV, Antelmann H, and Helmann JD. The role of bacillithiol in gram-positive firmicutes. Antioxid Redox Signal 28: 445-462, 2018.

14. Chen PR, Bae T, Williams WA, Duguid EM, Rice PA, Schneewind $\mathrm{O}$, and $\mathrm{He} \mathrm{C}$. An oxidation-sensing mechanism is used by the global regulator MgrA in Staphylococcus aureus. Nat Chem Biol 2: 591-595, 2006.

15. Chen PR, Brugarolas P, and He C. Redox signaling in human pathogens. Antioxid Redox Signal 14: 1107-1118, 2011.

16. Chen PR, Nishida S, Poor CB, Cheng A, Bae T, Kuechenmeister L, Dunman PM, Missiakas D, and He C. A new oxidative sensing and regulation pathway mediated by the MgrA homologue SarZ in Staphylococcus aureus. Mol Microbiol 71: 198-211, 2009.

17. Cheung AL, Nishina KA, Trotonda MP, and Tamber S. The SarA protein family of Staphylococcus aureus. Int J Biochem Cell Biol 40: 355-361, 2008.

18. Chi BK, Albrecht D, Gronau K, Becher D, Hecker M, and Antelmann H. The redox-sensing regulator YodB senses quinones and diamide via a thiol-disulfide switch in $B a$ cillus subtilis. Proteomics 10: 3155-3164, 2010.

19. Chi BK, Busche T, Van Laer K, Bäsell K, Becher D, Clermont L, Seibold GM, Persicke M, Kalinowski J, Messens J, and Antelmann H. Protein S-mycothiolation functions as redox-switch and thiol protection mechanism in Corynebacterium glutamicum under hypochlorite stress. Antioxid Redox Signal 20: 589-605, 2014.

20. Chi BK, Gronau K, Mader U, Hessling B, Becher D, and Antelmann H. S-bacillithiolation protects against hypochlorite stress in Bacillus subtilis as revealed by transcriptomics and redox proteomics. Mol Cell Proteomics 10: M111 009506, 2011.

21. Chi BK, Kobayashi K, Albrecht D, Hecker M, and Antelmann H. The paralogous MarR/DUF24-family repressors YodB and CatR control expression of the catechol dioxygenase CatE in Bacillus subtilis. J Bacteriol 192: 45714581, 2010.

22. Chi BK, Roberts AA, Huyen TT, Bäsell K, Becher D, Albrecht D, Hamilton CJ, and Antelmann H. S-bacillithiolation protects conserved and essential proteins against hypochlorite stress in firmicutes bacteria. Antioxid Redox Signal 18: 1273-1295, 2013.

23. Crack JC, Munnoch J, Dodd EL, Knowles F, Al Bassam MM, Kamali S, Holland AA, Cramer SP, Hamilton CJ, Johnson MK, Thomson AJ, Hutchings MI, and Le Brun NE. NsrR from Streptomyces coelicolor is a nitric oxide-sensing $[4 \mathrm{Fe}-4 \mathrm{~S}]$ cluster protein with a specialized regulatory function. J Biol Chem 290: 12689-12704, 2015.

24. Dal Peraro M and van der Goot FG. Pore-forming toxins: ancient, but never really out of fashion. Nat Rev Microbiol 14: 77-92, 2016.

25. Diep BA, Gill SR, Chang RF, Phan TH, Chen JH, Davidson MG, Lin F, Lin J, Carleton HA, Mongodin EF, Sensabaugh GF, and Perdreau-Remington F. Complete genome sequence of USA300, an epidemic clone of communityacquired meticillin-resistant Staphylococcus aureus. Lancet 367: 731-739, 2006.

26. Dubbs JM and Mongkolsuk S. Peroxiredoxins in bacterial antioxidant defense. Subcell Biochem 44: 143-193, 2007. 
27. Dubbs JM and Mongkolsuk S. Peroxide-sensing transcriptional regulators in bacteria. J Bacteriol 194: 5495-5503, 2012.

28. Even S, Burguiere P, Auger S, Soutourina O, Danchin A, and Martin-Verstraete I. Global control of cysteine metabolism by CymR in Bacillus subtilis. J Bacteriol 188: 2184-2197, 2006.

29. Falord M, Mader U, Hiron A, Debarbouille M, and Msadek T. Investigation of the Staphylococcus aureus GraSR regulon reveals novel links to virulence, stress response and cell wall signal transduction pathways. PLoS One 6: e21323, 2011.

30. Fomenko DE and Gladyshev VN. Comparative genomics of thiol oxidoreductases reveals widespread and essential functions of thiol-based redox control of cellular processes. Antioxid Redox Signal 16: 193-201, 2012.

31. Foster TJ. The Staphylococcus aureus "superbug". J Clin Invest 114: 1693-1696, 2004.

32. Fuangthong $M$, Atichartpongkul S, Mongkolsuk S, and Helmann JD. OhrR is a repressor of ohrA, a key organic hydroperoxide resistance determinant in Bacillus subtilis. $J$ Bacteriol 183: 4134-4141, 2001.

33. Gaballa A, Antelmann H, Hamilton CJ, and Helmann JD. Regulation of Bacillus subtilis bacillithiol biosynthesis operons by Spx. Microbiology 159: 2025-2035, 2013.

34. Gray MJ, Li Y, Leichert LI, Xu Z, and Jakob U. Does the transcription factor NemR use a regulatory sulfenamide bond to sense bleach? Antioxid Redox Signal 23: 747-754, 2015.

35. Gray MJ, Wholey WY, and Jakob U. Bacterial responses to reactive chlorine species. Annu Rev Microbiol 67: 141-160, 2013.

36. Gray MJ, Wholey WY, Parker BW, Kim M, and Jakob U. NemR is a bleach-sensing transcription factor. J Biol Chem 288: 13789-13798, 2013.

37. Guerra FE, Addison CB, de Jong NW, Azzolino J, Pallister KB, van Strijp JA, and Voyich JM. Staphylococcus aureus SaeR/S-regulated factors reduce human neutrophil reactive oxygen species production. J Leukoc Biol 100: 1005-1010, 2016.

38. Guo H, Hall JW, Yang J, and Ji Y. The SaeRS twocomponent system controls survival of Staphylococcus aureus in human blood through regulation of coagulase. Front Cell Infect Microbiol 7: 204, 2017.

39. Hampton MB, Kettle AJ, and Winterbourn CC. Involvement of superoxide and myeloperoxidase in oxygendependent killing of Staphylococcus aureus by neutrophils. Infect Immun 64: 3512-3517, 1996.

40. Hibbing ME and Fuqua C. Antiparallel and interlinked control of cellular iron levels by the Irr and RirA regulators of Agrobacterium tumefaciens. J Bacteriol 193: 34613472, 2011.

41. Highlander SK, Hulten KG, Qin X, Jiang H, Yerrapragada S, Mason EO, Jr., Shang Y, Williams TM, Fortunov RM, Liu Y, Igboeli O, Petrosino J, Tirumalai M, Uzman A, Fox GE, Cardenas AM, Muzny DM, Hemphill L, Ding Y, Dugan S, Blyth PR, Buhay CJ, Dinh HH, Hawes AC, Holder M, Kovar CL, Lee SL, Liu W, Nazareth LV, Wang Q, Zhou J, Kaplan SL, and Weinstock GM. Subtle genetic changes enhance virulence of methicillin resistant and sensitive Staphylococcus aureus. BMC Microbiol 7: 99, 2007.

42. Hilker R, Stadermann KB, Doppmeier D, Kalinowski J, Stoye J, Straube J, Winnebald J, and Goesmann A.
ReadXplorer-visualization and analysis of mapped sequences. Bioinformatics 30: 2247-2254, 2014.

43. Hillion M and Antelmann H. Thiol-based redox switches in prokaryotes. Biol Chem 396: 415-444, 2015.

44. Hillion M, Bernhardt J, Busche T, Rossius M, Maass S, Becher D, Rawat M, Wirtz M, Hell R, Ruckert C, Kalinowski J, and Antelmann H. Monitoring global protein thiol-oxidation and protein S-mycothiolation in Mycobacterium smegmatis under hypochlorite stress. Sci Rep 7: 1195, 2017.

45. Ilbert M, Graf PC, and Jakob U. Zinc center as redox switch-new function for an old motif. Antioxid Redox Signal 8: 835-846, 2006.

46. Imber M, Huyen NTT, Pietrzyk-Brzezinska AJ, Loi VV, Hillion M, Bernhardt J, Thärichen L, Kolsek K, Saleh M, Hamilton CJ, Adrian L, Gräter F, Wahl MC, and Antelmann H. Protein S-bacillithiolation functions in thiol protection and redox regulation of the glyceraldehyde-3phosphate dehydrogenase gap in Staphylococcus aureus under hypochlorite stress. Antioxid Redox Signal 28: 410430, 2018.

47. Ji Q, Zhang L, Sun F, Deng X, Liang H, Bae T, and He C. Staphylococcus aureus CymR is a new thiol-based oxidation-sensing regulator of stress resistance and oxidative response. J Biol Chem 287: 21102-21109, 2012.

48. Klebanoff SJ, Kettle AJ, Rosen H, Winterbourn CC, and Nauseef WM. Myeloperoxidase: a front-line defender against phagocytosed microorganisms. J Leukoc Biol 93: 185-198, 2013

49. Kosower NS and Kosower EM. Diamide: an oxidant probe for thiols. Methods Enzymol 251: 123-133, 1995.

50. Lee C, Shin J, and Park C. Novel regulatory system nemRA-gloA for electrophile reduction in Escherichia coli K-12. Mol Microbiol 88: 395-412, 2013.

51. Lee JW, Soonsanga S, and Helmann JD. A complex thiolate switch regulates the Bacillus subtilis organic peroxide sensor OhrR. Proc Natl Acad Sci U S A 104: 8743-8748, 2007.

52. Leelakriangsak M, Huyen NT, Töwe S, van Duy N, Becher D, Hecker M, Antelmann H, and Zuber P. Regulation of quinone detoxification by the thiol stress sensing DUF24/ MarR-like repressor, YodB in Bacillus subtilis. Mol Microbiol 67: 1108-1124, 2008.

53. Leichert LI and Jakob U. Global methods to monitor the thiol-disulfide state of proteins in vivo. Antioxid Redox Signal 8: 763-772, 2006.

54. Li S, Li P, Zhang L, Hu W, Wang M, Liu Y, Tang G, Wang $\mathrm{D}$, Zhou B, and Yan J. The role of reactive oxygen intermediates in the intracellular fate of Leptospira interrogans in the macrophages of different hosts. PLoS One 12: $\mathrm{e} 0178618,2017$.

55. Liu P, Wu X, Liao C, Liu X, Du J, Shi H, Wang X, Bai X, Peng P, Yu L, Wang F, Zhao Y, and Liu M. Escherichia coli and Candida albicans induced macrophage extracellular trap-like structures with limited microbicidal activity. PLoS One 9: e90042, 2014.

56. Liu Q, Yeo WS, and Bae T. The SaeRS two-component system of Staphylococcus aureus. Genes (Basel) 7: 81, 2016.

57. Livermore DM. Antibiotic resistance in staphylococci. Int $J$ Antimicrob Agents 16 Suppl 1: S3-S10, 2000.

58. Loi VV, Harms M, Müller M, Huyen NTT, Hamilton CJ, Hochgräfe F, Pane-Farre J, and Antelmann H. Real-time imaging of the bacillithiol redox potential in the human pathogen Staphylococcus aureus using a genetically 
encoded bacilliredoxin-fused redox biosensor. Antioxid Redox Signal 26: 835-848, 2017.

59. Love MI, Huber W, and Anders S. Moderated estimation of fold change and dispersion for RNA-seq data with DESeq2. Genome Biol 15: 550, 2014.

60. Lowy FD. Staphylococcus aureus infections. $N$ Engl J Med 339: 520-532, 1998.

61. Mäder U, Nicolas P, Depke M, Pane-Farre J, Debarbouille M, van der Kooi-Pol MM, Guerin C, Derozier S, Hiron A, Jarmer H, Leduc A, Michalik S, Reilman E, Schaffer M, Schmidt F, Bessieres P, Noirot P, Hecker M, Msadek T, Volker U, and van Dijl JM. Staphylococcus aureus transcriptome architecture: from laboratory to infectionmimicking conditions. PLoS Genet 12: e1005962, 2016.

62. Mehlan H, Schmidt F, Weiss S, Schüler J, Fuchs S, Riedel $\mathrm{K}$, and Bernhardt J. Data visualization in environmental proteomics. Proteomics 13: 2805-2821, 2013.

63. Moore MJ, Miller SM, and Walsh CT. C-terminal cysteines of Tn501 mercuric ion reductase. Biochemistry 31: 16771685, 1992.

64. Moore MJ and Walsh CT. Mutagenesis of the N- and Cterminal cysteine pairs of Tn501 mercuric ion reductase: consequences for bacterial detoxification of mercurials. Biochemistry 28: 1183-1194, 1989.

65. More P and Pai K. In vitro NADH-oxidase, NADPH-oxidase and myeloperoxidase activity of macrophages after Tinospora cordifolia (guduchi) treatment. Immunopharmacol Immunotoxicol 34: 368-372, 2012.

66. Müller M, Reiss S, Schlüter R, Mader U, Beyer A, Reiss W, Marles-Wright J, Lewis RJ, Pförtner H, Völker U, Riedel K, Hecker M, Engelmann S, and Pane-Farre J. Deletion of membrane-associated Asp23 leads to upregulation of cell wall stress genes in Staphylococcus aureus. Mol Microbiol 93: 1259-1268, 2014.

67. Munnoch JT, Martinez MT, Svistunenko DA, Crack JC, Le Brun NE, and Hutchings MI. Characterization of a putative NsrR homologue in Streptomyces venezuelae reveals a new member of the Rrf2 superfamily. Sci Rep 6: 31597, 2016.

68. Nakano MM, Kominos-Marvell W, Sane B, Nader YM, Barendt SM, Jones MB, and Zuber P. spxA2, encoding a regulator of stress resistance in Bacillus anthracis, is controlled by SaiR, a new member of the Rrf2 protein family. Mol Microbiol 94: 815-827, 2014.

69. Nakano S, Kuster-Schock E, Grossman AD, and Zuber P. Spx-dependent global transcriptional control is induced by thiol-specific oxidative stress in Bacillus subtilis. Proc Natl Acad Sci U S A 100: 13603-13608, 2003.

70. Newberry KJ, Nakano S, Zuber P, and Brennan RG. Crystal structure of the Bacillus subtilis anti-alpha, global transcriptional regulator, Spx, in complex with the alpha Cterminal domain of RNA polymerase. Proc Natl Acad Sci U S A 102: 15839-15844, 2005.

71. Nicolas P, Mäder U, Dervyn E, Rochat T, Leduc A, Pigeonneau N, Bidnenko E, Marchadier E, Hoebeke M, Aymerich S, Becher D, Bisicchia P, Botella E, Delumeau O, Doherty G, Denham EL, Fogg MJ, Fromion V, Goelzer A, Hansen A, Hartig E, Harwood CR, Homuth G, Jarmer H, Jules M, Klipp E, Le Chat L, Lecointe F, Lewis P, Liebermeister W, March A, Mars RA, Nannapaneni P, Noone D, Pohl S, Rinn B, Rugheimer F, Sappa PK, Samson F, Schaffer M, Schwikowski B, Steil L, Stülke J, Wiegert T, Devine KM, Wilkinson AJ, van Dijl JM, Hecker M, Völker U, Bessieres P, and Noirot P. Condition-dependent transcriptome reveals high-level regulatory architecture in Bacillus subtilis. Science 335: 1103-1106, 2012.

72. Ozyamak E, de Almeida C, de Moura AP, Miller S, and Booth IR. Integrated stress response of Escherichia coli to methylglyoxal: transcriptional readthrough from the nemRA operon enhances protection through increased expression of glyoxalase I. Mol Microbiol 88: 936-950, 2013.

73. Palm GJ, Khanh Chi B, Waack P, Gronau K, Becher D, Albrecht D, Hinrichs W, Read RJ, and Antelmann H. Structural insights into the redox-switch mechanism of the MarR/DUF24-type regulator HypR. Nucleic Acids Res 40: 4178-4192, 2012.

74. Panmanee W, Vattanaviboon P, Eiamphungporn W, Whangsuk W, Sallabhan R, and Mongkolsuk S. OhrR, a transcription repressor that senses and responds to changes in organic peroxide levels in Xanthomonas campestris pv. phaseoli. Mol Microbiol 45: 1647-1654, 2002.

75. Parker BW, Schwessinger EA, Jakob U, and Gray MJ. The $\mathrm{RclR}$ protein is a reactive chlorine-specific transcription factor in Escherichia coli. J Biol Chem 288: 32574-32584, 2013.

76. Pendleton JN, Gorman SP, and Gilmore BF. Clinical relevance of the ESKAPE pathogens. Expert Rev Anti Infect Ther 11: 297-308, 2013.

77. Pförtner H, Burian MS, Michalik S, Depke M, Hildebrandt P, Dhople VM, Pane-Farre J, Hecker M, Schmidt F, and Völker U. Activation of the alternative sigma factor SigB of Staphylococcus aureus following internalization by epithelial cells - an in vivo proteomics perspective. Int J Med Microbiol 304: 177-187, 2014.

78. Poor CB, Chen PR, Duguid E, Rice PA, and He C. Crystal structures of the reduced, sulfenic acid, and mixed disulfide forms of SarZ, a redox active global regulator in Staphylococcus aureus. J Biol Chem 284: 23517-23524, 2009.

79. Pöther DC, Gierok P, Harms M, Mostertz J, Hochgräfe F, Antelmann H, Hamilton CJ, Borovok I, Lalk M, Aharonowitz $\mathrm{Y}$, and Hecker M. Distribution and infectionrelated functions of bacillithiol in Staphylococcus aureus. Int J Med Microbiol 303: 114-123, 2013.

80. Ralph P, Moore MA, and Nilsson K. Lysozyme synthesis by established human and murine histiocytic lymphoma cell lines. J Exp Med 143: 1528-1533, 1976.

81. Rosenblum ED and Tyrone S. Serology, density, and morphology of Staphylococcal phages. J Bacteriol 88: 1737-1742, 1964.

82. Santos JA, Pereira PJ, and Macedo-Ribeiro S. What a difference a cluster makes: the multifaceted roles of IscR in gene regulation and DNA recognition. Biochim Biophys Acta 1854: 1101-1112, 2015.

83. Schwartz CJ, Giel JL, Patschkowski T, Luther C, Ruzicka FJ, Beinert $\mathrm{H}$, and Kiley PJ. IscR, an Fe-S clustercontaining transcription factor, represses expression of Escherichia coli genes encoding Fe-S cluster assembly proteins. Proc Natl Acad Sci U S A 98: 14895-14900, 2001.

84. Shepard W, Soutourina O, Courtois E, England P, Haouz A, and Martin-Verstraete I. Insights into the Rrf2 repressor family-the structure of CymR, the global cysteine regulator of Bacillus subtilis. FEBS J 278: 2689-2701, 2011.

85. Spaan AN, van Strijp JAG, and Torres VJ. Leukocidins: staphylococcal bi-component pore-forming toxins find their receptors. Nat Rev Microbiol 15: 435-447, 2017. 
86. Sreerama N and Woody RW. Estimation of protein secondary structure from circular dichroism spectra: comparison of CONTIN, SELCON, and CDSSTR methods with an expanded reference set. Anal Biochem 287: 252260, 2000.

87. Suckau D, Resemann A, Schuerenberg M, Hufnagel P, Franzen J, and Holle A. A novel MALDI LIFT-TOF/TOF mass spectrometer for proteomics. Anal Bioanal Chem 376: 952-965, 2003.

88. Sun F, Ding Y, Ji Q, Liang Z, Deng X, Wong CC, Yi C, Zhang L, Xie S, Alvarez S, Hicks LM, Luo C, Jiang H, Lan $\mathrm{L}$, and He C. Protein cysteine phosphorylation of SarA/ MgrA family transcriptional regulators mediates bacterial virulence and antibiotic resistance. Proc Natl Acad Sci U S A 109: 15461-15466, 2012.

89. Tam le T, Eymann C, Albrecht D, Sietmann R, Schauer F, Hecker M, and Antelmann H. Differential gene expression in response to phenol and catechol reveals different metabolic activities for the degradation of aromatic compounds in Bacillus subtilis. Environ Microbiol 8: 1408-1427, 2006.

90. Volbeda A, Dodd EL, Darnault C, Crack JC, Renoux O, Hutchings MI, Le Brun NE, and Fontecilla-Camps JC. Crystal structures of the NO sensor NsrR reveal how its iron-sulfur cluster modulates DNA binding. Nat Commun 8: 15052, 2017.

91. Voyich JM, Braughton KR, Sturdevant DE, Whitney AR, Said-Salim B, Porcella SF, Long RD, Dorward DW, Gardner DJ, Kreiswirth BN, Musser JM, and DeLeo FR. Insights into mechanisms used by Staphylococcus aureus to avoid destruction by human neutrophils. J Immunol 175: 3907-3919, 2005.

92. Voyich JM, Vuong C, DeWald M, Nygaard TK, Kocianova S, Griffith S, Jones J, Iverson C, Sturdevant DE, Braughton KR, Whitney AR, Otto M, and DeLeo FR. The SaeR/S gene regulatory system is essential for innate immune evasion by Staphylococcus aureus. J Infect Dis 199: 16981706, 2009.

93. Wetzstein M, Völker U, Dedio J, Lobau S, Zuber U, Schiesswohl M, Herget C, Hecker M, and Schumann W. Cloning, sequencing, and molecular analysis of the dnaK locus from Bacillus subtilis. J Bacteriol 174: 3300-3310, 1992.

94. Whitmore L and Wallace BA. Protein secondary structure analyses from circular dichroism spectroscopy: methods and reference databases. Biopolymers 89: 392-400, 2008.

95. Winter J, Ilbert M, Graf PC, Ozcelik D, and Jakob U. Bleach activates a redox-regulated chaperone by oxidative protein unfolding. Cell 135: 691-701, 2008.

96. Winterbourn CC and Kettle AJ. Redox reactions and microbial killing in the neutrophil phagosome. Antioxid Redox Signal 18: 642-660, 2013.

97. Winterbourn CC, Kettle AJ, and Hampton MB. Reactive oxygen species and neutrophil function. Annu Rev Biochem 85: 765-792, 2016

98. Yukl ET, Elbaz MA, Nakano MM, and Moenne-Loccoz P. Transcription factor NsrR from Bacillus subtilis senses nitric oxide with a $4 \mathrm{Fe}-4 \mathrm{~S}$ cluster (dagger). Biochemistry 47: 13084-13092, 2008.

99. Zuber P. Spx-RNA polymerase interaction and global transcriptional control during oxidative stress. J Bacteriol 186: 1911-1918, 2004.
Address correspondence to: Prof. Haike Antelmann Institute for Biology-Microbiology Freie Universität Berlin

Königin-Luise-Strasse 12-16

Berlin D-14195

Germany

E-mail: haike.antelmann@fu-berlin.de

Date of first submission to ARS Central, September 4, 2017; date of final revised submission, November 21, 2017; date of acceptance, December 9, 2017.

\begin{tabular}{|c|}
\hline 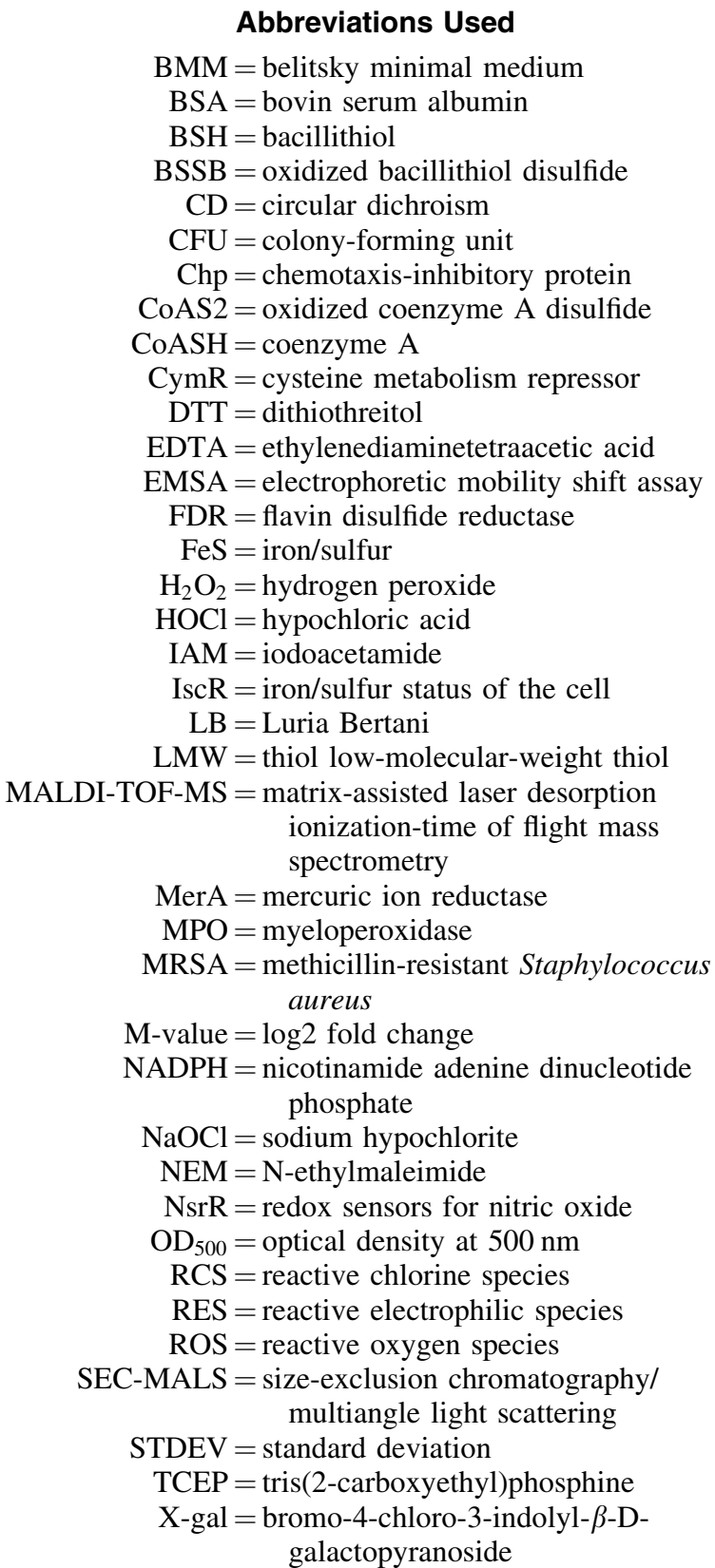 \\
\hline
\end{tabular}

